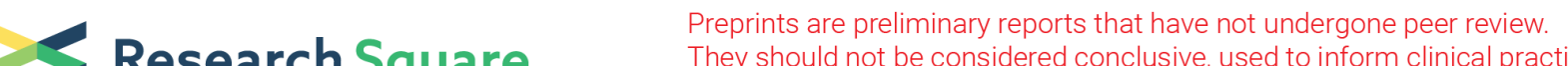 Research Square $\quad \begin{aligned} & \text { They should not be considered conclusive, used to inform clinical practice, } \\ & \text { or referenced by the media as validated information. }\end{aligned}$
}

\section{The Proteome, Not the Transcriptome, Predicts that Oocyte Superovulation Affects Embryonic Phenotypes in Mice}

\section{Leila Taher}

Graz University of Technology

\section{Steffen Israel}

Max Planck Institute for Molecular Biomedicine

Hannes Drexler

Max Planck Institute for Molecular Biomedicine

\section{Wojciech Makalowski}

University of Münster

\section{Yutaka Suzuki}

University of Tokyo

\section{Georg Fuellen}

Universitätsmedizin Rostock

Michele Boiani ( $\nabla$ mboiani@mpi-muenster.mpg.de )

Max Planck Institute for Molecular Biomedicine

\section{Research Article}

Keywords: Proteome, Transcriptome, Oocyte Superovulation, Embryonic Phenotypes

Posted Date: July 28th, 2021

DOl: https://doi.org/10.21203/rs.3.rs-724812/v1

License: (9) This work is licensed under a Creative Commons Attribution 4.0 International License.

Read Full License

Version of Record: A version of this preprint was published at Scientific Reports on December 1st, 2021. See the published version at https://doi.org/10.1038/s41598-021-03054-9. 


\section{The proteome, not the transcriptome, predicts that oocyte superovulation affects embryonic phenotypes in mice}

Authors: Leila Taher ${ }^{1, *}$, Steffen Israel ${ }^{2}$, Hannes C.A. Drexler ${ }^{2}$, Wojciech Makalowski ${ }^{4}$, Yutaka Suzuki ${ }^{5}$, Georg Fuellen ${ }^{3,},{ }^{*}$ Michele Boiani ${ }^{2,}{ }^{*}$

${ }^{1}$ Graz University of Technology, Institute of Biomedical Informatics, Stremayrgasse 16/I, A-8010 Graz

${ }^{2}$ Max Planck Institute for Molecular Biomedicine, Roentgenstrasse 20, 48149 Muenster, Germany

${ }^{3}$ Rostock University Medical Center, Institute for Biostatistics and Informatics in Medicine and Aging Research (IBIMA), Ernst-Heydemann-Strasse 8, 18057 Rostock, Germany

${ }^{4}$ Institute of Bioinformatics, Faculty of Medicine, University of Münster, Niels Stensen Str. 14, 48149, Münster, Germany.

${ }^{5}$ Department of Medical Genome Sciences, Graduate School of Frontier Sciences, University of Tokyo, Kashiwa, Chiba, 277-8562, Japan.

*: shared corresponding authors: L.T., leila.taher@tugraz.at

G.F., fuellen@nuni-rostock.de

M.B., mboiani@mpi-muenster.mpg.de

Main contact during the review process: Michele Boiani, mboiani@mpi-muenster.mpg.de 


\section{Abstract}

Superovulation is the epitome for generating oocytes for molecular embryology in mice, and it is used to model medically assisted reproduction in humans. Yet whether a superovulated oocyte is normal, is an open question. This study establishes for the first time that superovulation is associated with proteome changes that bear on phenotypic traits in mice, whereas the transcriptome is far less predictive. The proteins that were differentially expressed in superovulated mouse oocytes and their derived embryos relative to their naturally ovulated counterparts were enriched in ontology terms describing abnormal mammalian phenotypes: a thinner zona pellucida, a smaller oocyte diameter, increased cleavage arrest, and defective blastocyst formation, which we could all verify functionally. Moreover, our findings indicate that embryos with such abnormalities are negatively selected during preimplantation, and ascribe these abnormalities to incomplete ovarian maturation during the time of the conventional superovulation, since they could be corrected upon postponement of the ovulatory stimulus by $24 \mathrm{~h}$. Our data place constraints on the common view that superovulated oocytes are suited to draw conclusions of general validity about developmental processes, and underscore the importance of including the proteins in a modern molecular definition of oocyte quality. 


\section{Introduction}

The term 'superovulation' was introduced in $1927^{1,2}$ and has become the epitome for generating oocytes for experimental and molecular embryology in mice, in greater numbers than natural ovulation, by stimulation with exogenous gonadotropins. Studies on both superovulation and pregnancy in mice set the stage for human assisted reproduction ${ }^{3-5}$. Now as back then, the superovulation of mice relies on the administration of equine and human chorionic gonadotropin, eCG and hCG, $48 \mathrm{~h}$ or approximately two days apart ${ }^{6}$. There has been a myriad of studies to illuminate developmental processes using superovulated oocytes, in lieu of naturally ovulated oocytes. One could almost say provocatively that molecular mouse embryology is an embryology of superovulation. The reason for preferring the superovulated oocytes is one of convenience: fewer donor animals are needed, and the time of oocyte collection can be scheduled. The studies suggested that the superovulated oocytes were either equivalent or that they were not equivalent to naturally ovulated oocytes. These conflicting conclusions were based on heterogeneous epigenetic data, and rare transcriptomic data. We hypothesized that proteomic data may provide a more robust answer, since gene transcription is silenced in late oogenesis, and the initial stages of embryogenesis are controlled by maternal proteins ${ }^{7-9}$.

Oocyte quality aberrations associated with the gonadotropins eCG and hCG were not equally observed across studies. Superovulated mouse oocytes had reduced ability for endocytosis ${ }^{10}$ and derivative blastocysts had fewer microvilli ${ }^{11}$ than naturally ovulated counterparts, but ultrastructural differences were otherwise unimpressive. Some studies reported an increase of the rate of embryo aneuploidy and fetal malformation by use of gonadotropins ${ }^{12-16}$ while others contradicted these findings ${ }^{4,17,18}$. Likewise, while some studies linked the superovulation to a direct effect on the acquisition of DNA methylation in oocytes ${ }^{19-23}$, including epimutations ${ }^{24}$, other studies found no such effect ${ }^{25,26}$. In particular, one study suggested that superovulation results in immature oocytes that have not completed the acquisition of DNA methylation ${ }^{27}$, whereas another study found that oocyte diameters - which correlate with oocyte maturity and DNA methylation ${ }^{28}$ - do not differ between superovulation and natural ovulation ${ }^{26}$. In most of the studies above, there was a 
common confounder: the time spent by the oocytes in the genital tract of the stimulated female (interval between superovulation and collection for study). There has been a concern that superovulation may create an unfavorable somatic (endocrine) environment for the developing embryo either in the oviduct ${ }^{29,30}$ or in the uterus ${ }^{31-37}$; the latter would lead to reduced receptivity and thereby reduced blastocyst implantation, with later consequences for placental function ${ }^{38}$. Unlike the interval between superovulation and oviductal retrieval, the interval between eCG and hCG has been neglected in mice, with only two studies that reached different conclusions ${ }^{39,40}$. Yet it goes by itself that the accumulation of gene products in oocytes is a function of time during oogenesis (including time between eCG and hCG). Therefore, it is conceivable that the variable results listed above were contributed, at least in part, by a variable extent of oocyte's maturation prior to the administration of hCG.

The overarching aim of our study was to clarify the mechanism of the superovulation effect on oocyte quality in mice, drawing from a type of data not previously used in a context of superovulation vs. natural ovulation. No tandem studies of transcriptome and proteome have been yet devoted to the comparison between the gene expression profiles of superovulated and naturally ovulated mouse oocytes and derivative embryos, in a way that the two groups differ solely by the superovulation. Unlike the extensive use of transcriptome analysis in cell biology, this approach has only been used once to compare superovulated and naturally ovulated mouse oocytes ${ }^{41}$, with inconspicuous findings. There have been hints that protein synthesis in oocytes ${ }^{42,43}$ and maternal proteins required for imprint maintenance after fertilization ${ }^{44}$ may be disturbed by gonadotropins, but these hints were not followed up. Building on our record of oocyte and embryo OMICS analysis ${ }^{45-}$ ${ }^{50}$ we conducted a comparative proteome vs. transcriptome analysis of the two groups. To this end, the in vivo-fertilized oocytes of this study were not left in the genital tract of the stimulated female for 1 to 3 days as per common practice ${ }^{38,51}$, but were removed immediately, cultured, and analyzed for gene expression or transferred to naturally cycling recipients to complete development. The predictions of the gene expression analysis were cross-checked with the occurrence of relevant phenotypes during pre- and postimplantation development. The results reported here establish that superovulated mouse oocytes are not equivalent to their natural counterparts: decisive is the extent of preovulatory development prior to the ovulatory stimulus, and the non-equivalence could not have been inferred from a transcriptome analysis. This underscores the importance of 
including the proteins in a modern molecular definition of oocyte quality. Since our protein analysis has revealed unsuspected effects of superovulation, our study calls for caution when relying on superovulated oocytes to draw conclusions of general validity about developmental processes. 


\section{Results}

\section{Differences between superovulated oocytes and embryos, and their naturally ovulated}

counterparts, are more pronounced in the proteome than in the transcriptome

We quantified and compared the proteome and the transcriptome of mouse oocytes and preimplantation embryos in two groups: i) 'superovulation' (treatment) and ii) 'natural ovulation' (control), without the developmental confounder of the maternal environment. In each of the two groups, we collected oocytes either before or after fertilization in vivo, and brought them on the same footing by culturing in $\mathrm{KSOM}(\mathrm{aa})$ medium (Figure 1), thereby enabling a follow-up of the fertilized oocytes during preimplantation (2-cell, 4-cell, 8-cell, morula, blastocyst; Supplementary Figure S1). For all developmental stages, we collected two biological replicates for proteomics and transcriptomics analyses. The in vitro blastocysts exhibited superior morphology compared to blastocysts collected in vivo, as exemplified by the degree of cavity expansion (Supplementary Figure S2). This difference attests to the influence of undefined cues emanating from the genital tract of stimulated females, and to the importance - in discovery studies - of eliminating the confounder of the maternal environment.

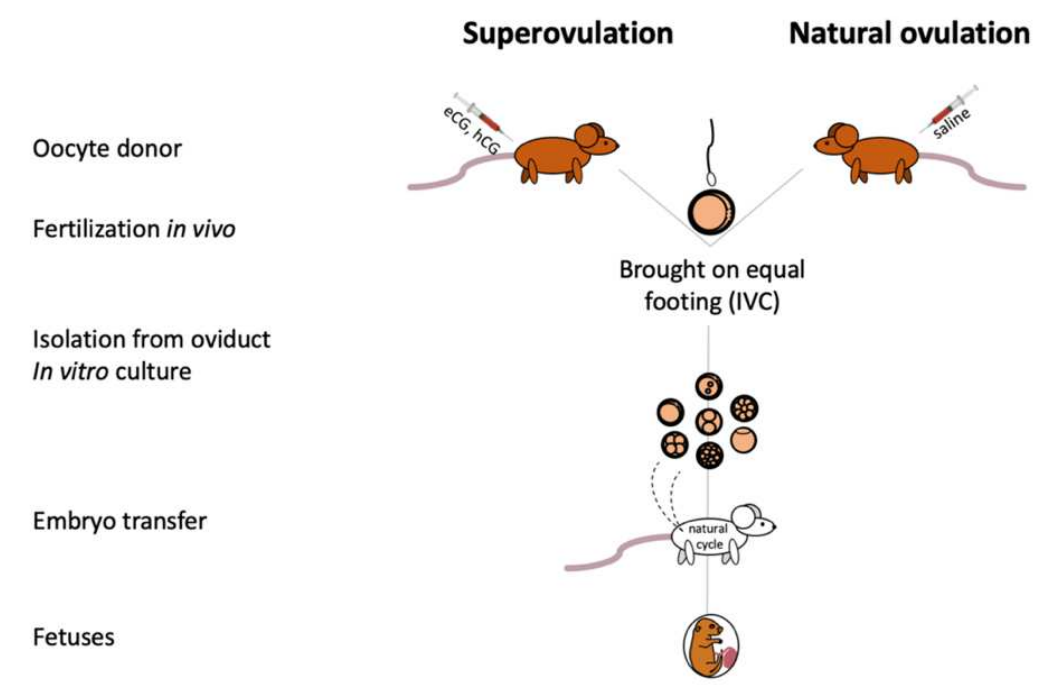

Figure 1. Experimental design. B6C3F1 females were injected with gonadotropins (superovulation) or with vehicle (natural ovulation) and caged with vasectomized or with stud CD1 males to obtain MII or fertilized oocytes, respectively. These were removed at 8 a.m. on the day of the vaginal plug, and brought on equal footing by in vitro culture (IVC). Embryos were sampled in vitro at defined stages for molecular analysis, or were transplanted to naturally cycling females to generate fetuses. Abbreviations: eCG, equine chorionic gonadotropin; hCG, human chorionic gonadotropin; IVC, in vitro culture, MII, metaphase II. 
Protein expression was measured by LC-MS/MS analysis of zona-free specimens as per our established analysis pipeline ${ }^{45-50}$. The zona-free cells were concentrated in a 2-3 $\mu \mathrm{L}$ volume of protein-free medium (Materials and Methods), as shown exemplarily for the oocytes (Supplementary Figure S1, 'pellet'), before adding $10 \mu \mathrm{L}$ of lysis buffer on top. Protein abundance was quantified using the riBAQ algorithm (Materials and Methods), which provides adimensional values that are proportional to the molar fraction of the proteins in the cell. We detected a total of 6,444 proteins across all samples (Supplementary Table S1). From the specimens collected for proteomics we set aliquots aside, which were subjected to RNA-seq and microarray analysis, returning a transcriptome of 20,887 transcripts (Supplementary Table S2).

Among the 6,444 proteins, 2,844 were detected in both treatment groups at all stages and in at least one replicate and were therefore considered "constitutively expressed" (Supplementary Table S3). Hierarchical clustering of the expression profile of these 2,844 proteins distinctly separated the samples into two clusters (Figure 2A): a cluster comprising the oocyte and 1-, 2-, 4-, and 8-cell stages, and a cluster comprising the morula and blastocyst stages. While in the latter the samples were clustered by stage, with superovulation and natural ovulation samples clustering together, the samples in the former were clustered according to a different pattern. Specifically, those samples were separated into four clusters consisting of exclusively superovulation or natural ovulation samples. This was in stark contrast to the hierarchical clustering of the corresponding transcripts, which showed all samples clustered by stage, with superovulation and natural ovulation samples clustering together (Figure 2E). These findings were robust, irrespective of whether we considered the set of constitutively expressed genes (Figure 2) or the parent sets of 6,444 proteins and 20,887 transcripts (Supplementary Figure S3). The hierarchical clustering results were consistent with principal component analysis (PCA). For the proteome, the first principal component (PC1, $27 \%$ of the variance) predominantly captured the developmental trajectory of the embryos (Figure 2B), confirming our previous study ${ }^{46}$. The second principal component (PC2, 14\% of the variance) distinguished between superovulation and natural ovulation oocytes and derivative embryos (Figure 2C). In contrast, the PCA of the transcriptome (Figure $2 \mathrm{~F}-\mathrm{H}$ ) revealed a much smaller effect of the treatment, which was only primarily captured by the sixth principal component (PC6, 1\% of the variance). 
These results reveal differences between the gene expression profiles of superovulated oocytes and derivative embryos and their naturally ovulated counterparts when compared on equal footing i.e. in the same in vitro environment. This molecular distinction is more pronounced in the proteome than in the transcriptome, delineating a gonadotropin-sensitive proteome. Since proteomics has not been used before to compare superovulation to natural ovulation, it follows that superovulation may affect developmental processes in unimagined ways.
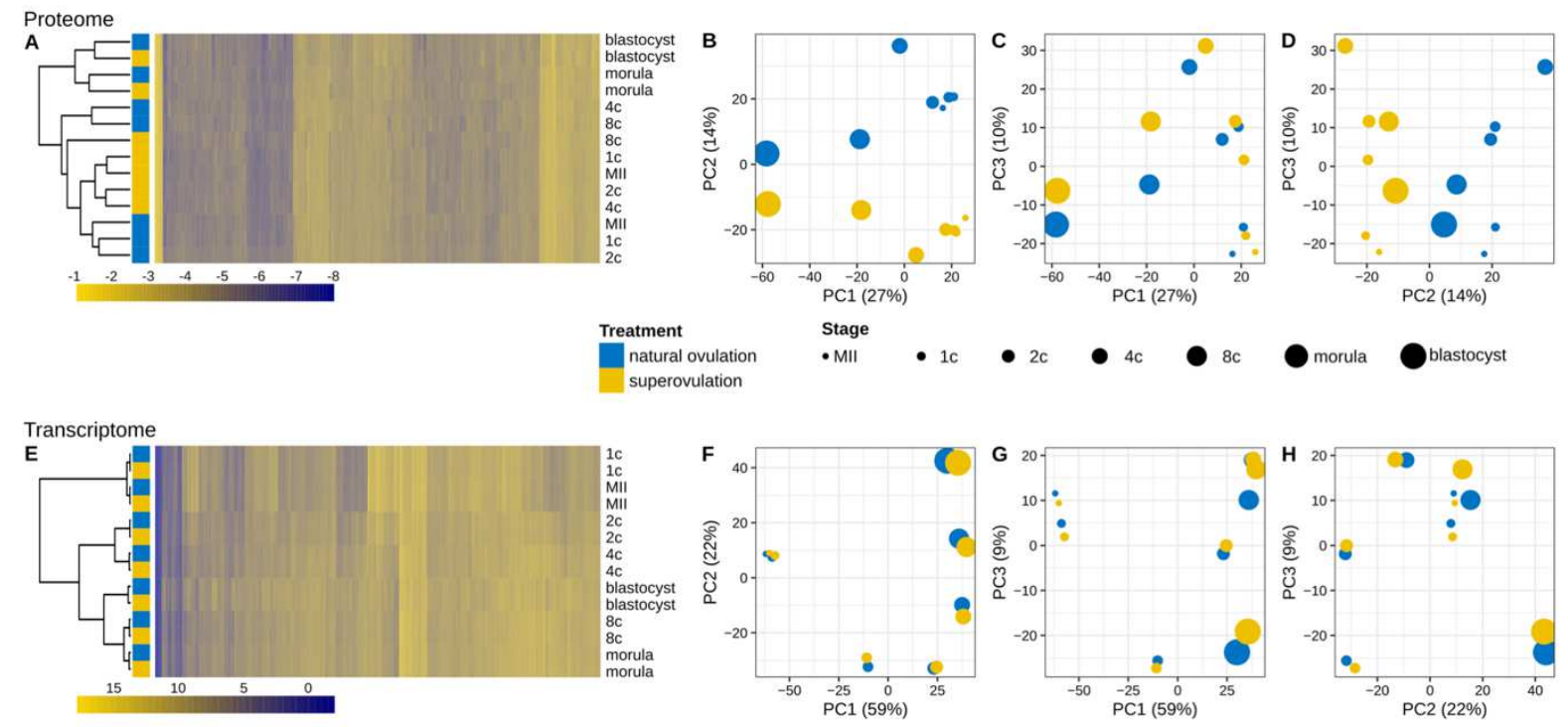

natural ovulation Stage - MII

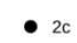

- $4 \mathrm{c}$

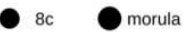

blastocyst
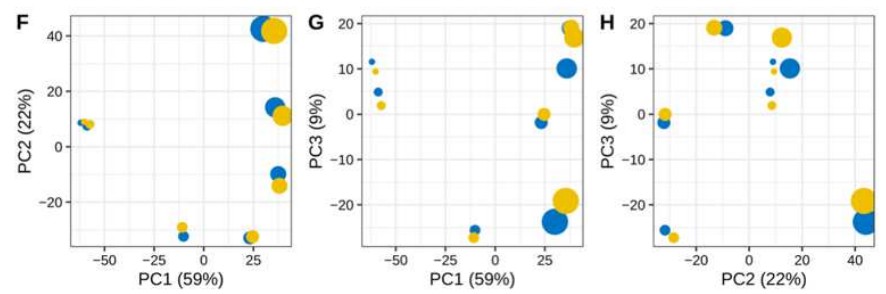

Figure 2. Expression analysis of the constitutively expressed $(n=2,844)$ proteome and their transcriptomic counterpart. (A, E) Hierarchical clustering of the proteome $(A)$ and the transcriptome (E). Samples were clustered with the complete linkage algorithm, using a distance matrix based on the Pearson correlation coefficient computed between the expression values of the proteins. Proteins/transcripts are sorted according to pairwise Euclidean distances. (B-D and F-H) Principal component analysis. Each data point corresponds to a stage and treatment group. The first three principal components (PCS) of the data are represented. The PCA of the proteome resolves the superovulation and the natural ovulation groups in the $2^{\text {nd }}$ component, whereas the PCA of the transcriptome resolves the two groups in the $6^{\text {th }}$ component (not shown). Samples were derived from pools of 200-250 (proteome) and 210 (transcriptome) oocytes or embryos, in duplicate for each treatment.

\section{A distinctive gene ontology signature in the altered proteome of superovulated oocytes}

To gain a general impression of how the gonadotropin-sensitive proteome fluctuates as a function of the developmental stage, we examined the fold-changes of the expression values of the 2,844 proteins between the superovulation and natural ovulation group. Specifically, 
we counted the number of proteins exceeding a particular fold-change (Figure 3A). Irrespective of the fold-change, the number of altered proteins had its maximum at the 8cell stage and its minimum in the blastocysts - consistent with a prior removal of the unfit or unviable embryos by natural selection. The overall profile suggests that specific proteins may be systematically up-regulated or systematically down-regulated across the entire developmental series in response to superovulation. We therefore examined this possibility.

A total of 278 of $2,844(10 \%)$ proteins were differentially expressed for all stages upon superovulation (Wilcoxon test; Supplementary Table S4; Materials and Methods). The abundance of these 278 proteins is systematically higher compared to that of the parent proteome, as judged from the probability density functions of riBAQ values (Supplementary Figure S4). Of the 278 proteins, 115 proteins were also differentially expressed in excess of 2-fold (56 up, 59 down, Supplementary Table S4).

Functional enrichment analysis of the 278 differentially expressed proteins revealed several terms in the Mammalian Phenotype Ontology (MP) that are relevant for pre- and post-implantation embryos and prompted additional experiments (see next section), including "MP:0009375 thin zona pellucida" (ZP), "MP:0001730 embryonic growth arrest", “MP:0012114 absent inner cell mass proliferation", and “MP:0011100 preweaning lethality, complete penetrance" (Figure 3B; Supplementary Table S4). The differentially expressed proteins also featured several terms in the gene ontology 'Biological process' (Figure 3C), with a stronger link to translation than to transcription. While this result may be unsurprising given the fact that we are analyzing proteomic data, it is also clear that protein activities enable transcription by e.g. RNA polymerases. The fact that RNA-related and transcriptionrelated functions are weakly represented among the differentially expressed proteins is entirely consistent with the results of the transcriptome analysis. Indeed, no transcripts were found differentially expressed (Supplementary Table S5), in contrast to the proteome. This was the case also for an additional transcriptomic dataset, which was based on a different platform (Affymetrix; GEO number GSE110599) but was derived from the same specimens used for the RNA-seq analysis. Thus, compared to their naturally ovulated counterparts, superovulated oocytes and embryos can be resolved the protein level much more so than at the transcript level. This is consistent with our previous findings ${ }^{46}$. 
To test the robustness of the gene ontology signature of the perturbed proteome, we next undertook a series of phenotypic analyses.

A

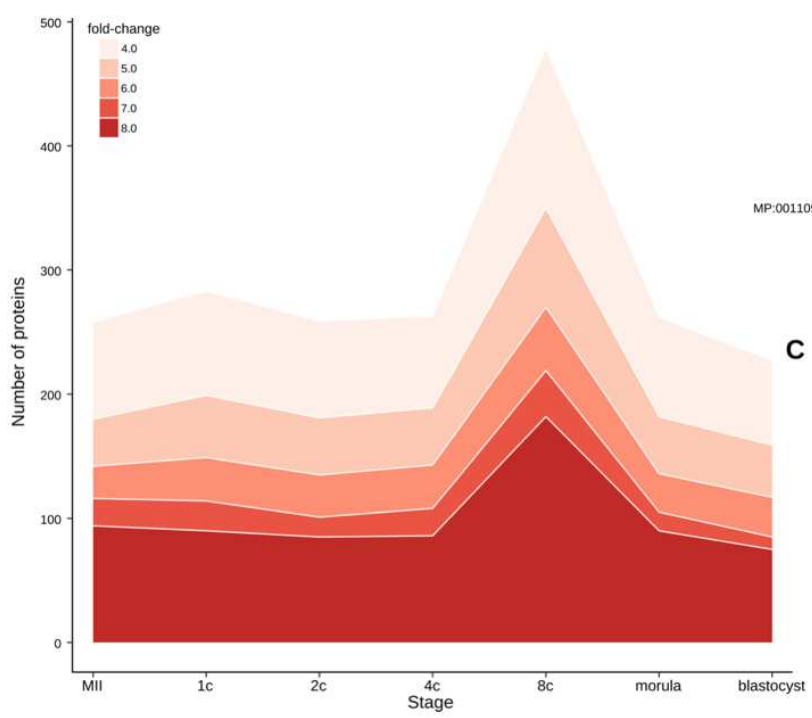

B

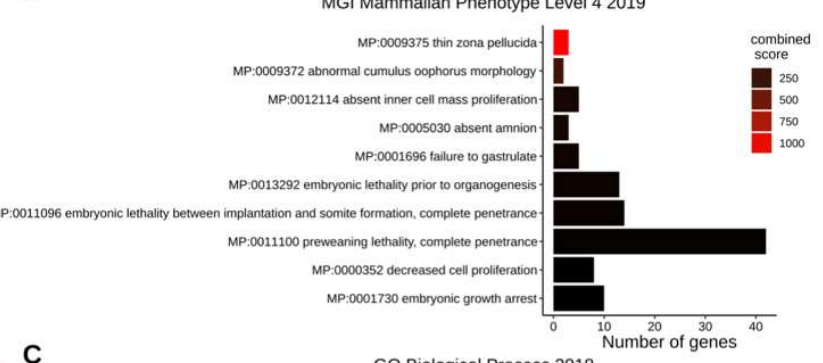

GO Biological Process 2018

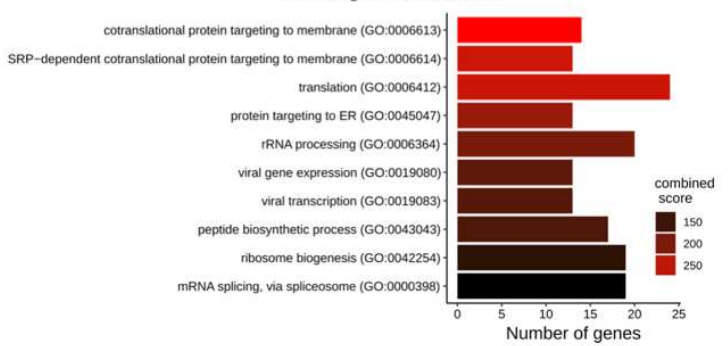

Figure 3. Numbers of gonadotropin-sensitive proteins and their functional enrichment analysis. (A) Stagespecific number of proteins exhibiting a fold-change greater than or equal to 4, 5, 6, 7, or 8 (see scale) between the superovulation and natural ovulation groups. (B, C) Functional enrichment analysis of differentially expressed proteins using Enrichr. Only the ten terms with the smallest P-values are shown. Terms are sorted according to their combined scores, which are computed by taking the logarithm of the P-values and multiplying them by the z-scores of the deviation from the expected ranks. (B) Gene ontology terms of the altered proteome in the ontology 'Mammalian Phenotype'. (C) Gene ontology terms of the altered proteome in the ontology 'Biological process'.

\section{Phenotypic traits substantiate the functional relevance of the altered proteome}

The functional enrichment analysis of the $\mathbf{2 7 8}$ differentially expressed proteins pointed at the top-term "thin zona pellucida" (ZP). This is represented in our dataset by the three proteins Zp1, Zp2, and Zp3, which account for the complete protein composition of the murine ZP. These proteins were systematically less abundant in superovulated oocytes and derivative embryos compared to their naturally ovulated counterparts. In contrast, no change was observed in the abundances of housekeeping genes (Supplementary Table S6). Differences in the abundances of ZP proteins might seem like an unexpected finding, given that the specimens of the proteomic analysis had been manually deprived of the ZP 
(Materials and Methods). However, it is pertinent to recall that ZP proteins are synthesized in the cytoplasm; thus, the alterations observed are presumably associated with cytoplasmic ZP proteins. To confirm this, we generated additional samples of ZP-free superovulated oocytes (Supplementary Figure S1) and compared them to ZP-enclosed oocytes via Western blot analysis, using a validated Zp3 antibody ${ }^{52}$ (Materials and Methods). This assay recognized a strong single band of the correct molecular weight regardless of whether the ZP had been removed or not, thereby showing that the bulk of Zp3 is intracellular (Supplementary Figure S1). ZP proteins are translocated into the endoplasmic reticulum, secreted, and assembled into fibrils outside the cell exclusively in growing oocytes. Therefore, our observations suggest that the oocytes had progressed unequally far into oogenesis when they were recruited for ovulation. In order to test this possibility, we left the ZP in place and measured its thickness and the diameter of the ooplasm (excluding the zona and perivitelline space) of freshly isolated, cumulus cell-free oocytes taken in parallel for the superovulation and natural ovulation groups (Materials and Methods). For brevity, the ooplasm without the ZP is also referred to as 'oocyte proper' or 'vitellus'. Indeed, superovulated oocytes exhibited a thinner ZP compared to their naturally ovulated counterparts (median 8.5 vs. $8.7 \mu \mathrm{m}$; interquartile range 8.1-8.8 $\mu \mathrm{m}$ vs. 8.3-9.1 $\mu \mathrm{m}$; P-value = 0.012, Wilcoxon test; Figure 4A). Moreover, since the oocyte diameter increases with the ZP thickness ${ }^{53}$, we inspected the diameter of the ooplasm. Since oocytes are approximately, but not exactly, spherical in shape, we derived the diameter from the perimeters drawn manually along the oolemma in the equatorial section of the oocytes (Materials and Methods). We found that the diameter of the oocyte proper was smaller for the superovulated oocytes compared to that of their naturally ovulated counterparts $(78.1 \pm 2.0$ $\mu \mathrm{m}$ vs. $79.4 \pm 2.0 \mu \mathrm{m}, \mathrm{p}<0.0001$, Wilcoxon test; Figure 4B). This dimensional difference in diameter accounts for a volume difference of $4.7 \%$, as calculated by applying spherical shape approximation (superovulated oocytes: 250 picoliters; naturally ovulated oocytes: 262 picoliters).

Another hit of the functional enrichment analysis, the MP term "embryonic growth arrest", was represented in our dataset by ten differentially expressed proteins (Emg1, Kdm1a, Hdac1, Cox17, Sumo2, Sbds, Cul1, Lamtor1, Pelo and Cops5; Supplementary Table S6). To functionally assess this term, we scored the proportions of embryos arrested at each preimplantation stage, starting from the 2-cell stage, so as to ensure that the oocyte had 
been fertilized (unfertilized oocytes would not cleave). The proportion of embryos arrested at or past the 2-cell stage is expected to be negligible, if the culture system is adequate (e.g. $\mathrm{KSOM}(\mathrm{aa}))$. Consistently, the vast majority of naturally ovulated oocytes progressed to blastocyst. By contrast, embryos of superovulated oocytes were more often arrested directly prior to blastocyst stage ( $P$-value $=0.013, \chi^{2}$ test; Figure $\left.4 C\right)$, with the greatest rate of loss at the morula stage. This is reminiscent of the observations made when embryos were flushed from the uterus after mating following to natural ovulation vs. superovulation: the former had the most losses at the beginning of development, while the latter had them toward the end of preimplantation and featured blastocysts of poor morphology (Supplementary Figure S2). Thus, unfit embryos of superovulated oocytes seemed to reach further i.e., to be selected later (morula-arrest) compared to natural ovulation (progression to blastocyst if not 2-cell arrest).

A third hit of the functional enrichment analysis, MP term "absent inner cell mass proliferation", was represented in our dataset by five differentially expressed proteins (Sbds, Pelo, Cdc73, Cops5 and Dab2; Supplementary Table S6). Since this term should reflect a direct impact on the number of cells found in the inner cell mass, and since proteins Dab2 and Cops 5 are lineage markers ${ }^{54}$, we counted those cells making a further distinction between the subcompartments of the inner cell mass (i.e., epiblast and primitive endoderm cells). As control, we counted the remaining blastocyst lineage, namely the trophectoderm. We relied on our immunostaining protocol to identify the cells in the three compartments (Figure 4D; Materials and Methods). The two subpopulations of the inner cell mass had less cells in the superovulation group (primitive endoderm, $\mathrm{P}$-value $=0.027$; epiblast, $\mathrm{P}$-value $=$ 0.004, Wilcoxon test; Figure 4E,F), while the trophectodermal and total cell counts were indistinguishable between blastocysts of the superovulation and natural ovulation groups (Figure 4G,H). Furthermore, the depletion was not merely a reduction, but it also featured cases of either no cells or just one cell in the endoderm compartment ( $P$-value $=0.002$; Fisher's exact test). These findings are informative, because the primitive endoderm and epiblast can interconvert via FGF signaling ${ }^{55}$, and a sufficient number of cells is needed in the epiblast in order for the blastocysts to be competent for fetal development ${ }^{56,57}$. It is therefore conceivable that cells might need to be present in sufficient numbers not only in the epiblast, but also in the primitive endoderm. Hence, we set out to examine how the embryos of the superovulation and natural ovulation group fare at postimplantation, which 
required embryo transfer (ET; Figure 5) so as to offer the same type of postimplantative environment to the two groups.

Following ET of $n=8$ embryos to pseudopregnant naturally cycling females (mated to a vasectomized male), the proportion of females that failed to become pregnant was skewed in the superovulation compared to the natural ovulation group ( 6 failures of 13 ETs after superovulation vs. 2 failures of 12 ETs after natural ovulation; see also the zero values for implantations and litters in Figure 5A,B), although this skew did not achieve significance for the sample size available ( $P$-value $=0,202$, Fisher's exact test). The number of fetuses recovered by hysterectomy on gestational day 18 and the number of implantations (fetuses + resorptions) were similar between the two groups (Figure 5A,B; Supplementary Figure S5), even when non-pregnant females were excluded from the analysis $(n=3.9 \pm 2.1$ vs. $n=4.0 \pm$ 2.1 in the superovulation group vs. natural ovulation group, respectively). Nevertheless, the fetuses of the superovulated group were significantly lighter than those of the natural ovulation group ( $\mathrm{P}$-value $=0.012$; Wilcoxon test; Figure $5 \mathrm{C}$ ); this was the case even if we distinguished between male and female fetuses, comparing males with males and females with females. Clearly, this observation is not due to sex ratio or uterine crowding, given the fixed number of 8 embryos transplanted. It is also not a consequence of low placental weight, which was conserved in the two groups (Figure 5D). Instead, this lighter body weight is reminiscent of the lower epiblast cell counts in the superovulated group (Figure 4E) and is consistent with the notion that the cells of the body stem from the epiblast. Apart from a lighter body weight, the pups did not present any obvious morphological anomaly.

Collectively, the above observations support that superovulated MII oocytes are smaller and their preimplantation embryos are developmentally inferior compared to their naturally ovulated counterparts. Embryos with a background of superovulation may also be at a disadvantage at implanting in the uterus. Past the hurdle of implantation though, development unfolds similarly in the two groups in terms of rates, although the fetuses of the superovulation group are lighter. 

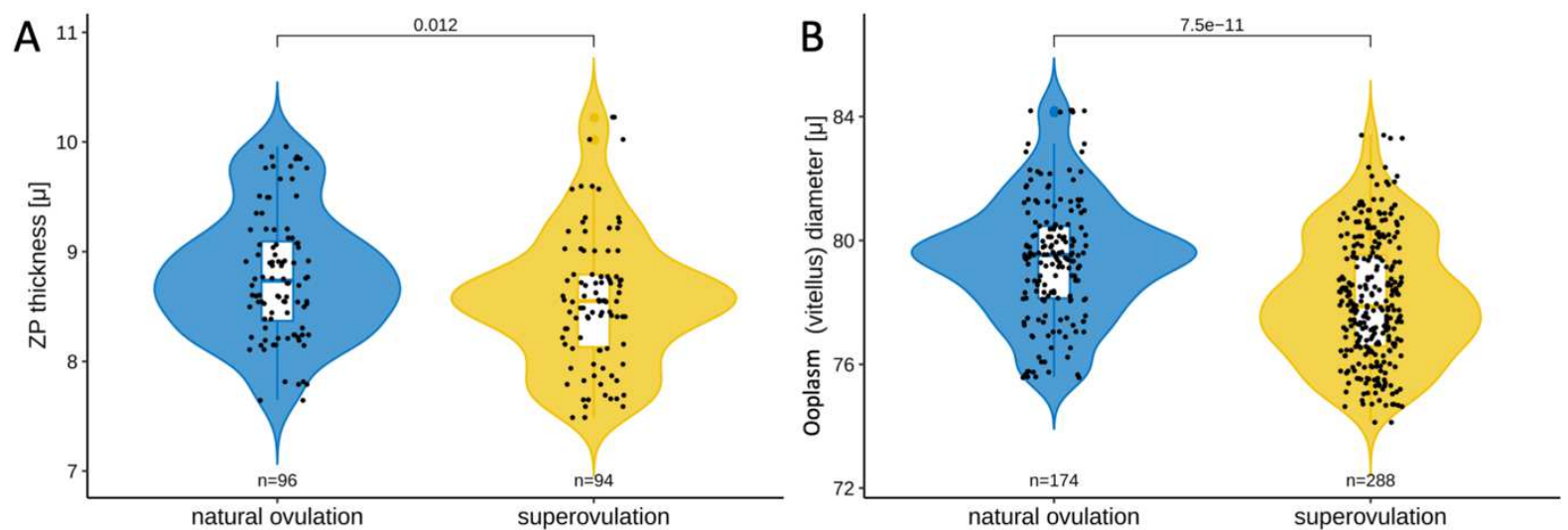

C

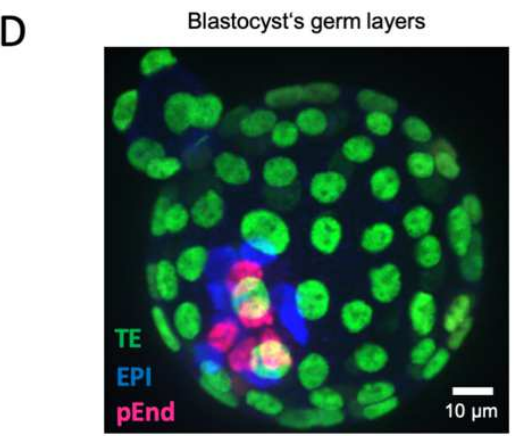

D
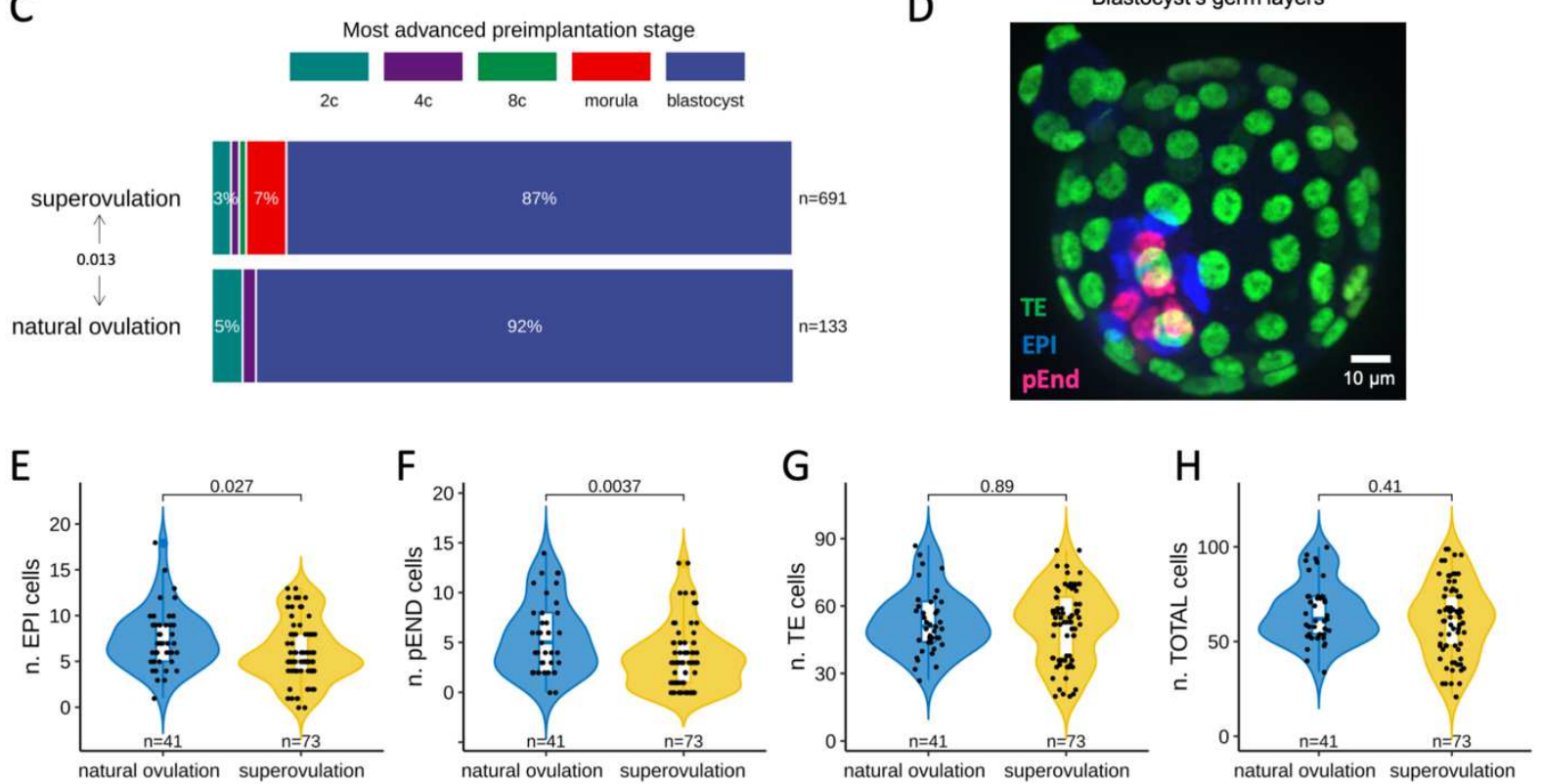

Figure 4. Preimplantation phenotypes of superovulated and naturally ovulated oocytes. (A) The thickness of the ZP. Each data point corresponds to one oocyte. Wilcoxon test. (B) The diameter of the oocyte proper i.e. excluding ZP and perivitelline space (vitellus). Each data point corresponds to one oocyte. Wilcoxon test. (C) The most advanced preimplantation stage reached in $\mathrm{KSOM}(\mathrm{aa})$ by the embryos of superovulated and naturally ovulated oocytes. Note that the scoring started from the 2-cell stage, since arrested 1-cells could be either oocytes which were not fertilized, or fertilized oocytes which failed to cleave. $\chi^{2}$ test. (D) Representative image of the triple immunostaining used to assign the blastocyst cells to the primary germ layers. $(\mathrm{E}-\mathrm{H})$ the cell numbers counted in the primary germ layers of blastocysts formed in $\mathrm{KSOM}(\mathrm{aa})$ by superovulated and naturally ovulated oocytes. Each data point corresponds to one blastocyst. Wilcoxon test. Abbreviations: $n$, number of; $\mathrm{TE}$, trophectoderm; EPI, epiblast; $\mathrm{pEND}$, primitive endoderm; ZP, zona pellucida. 

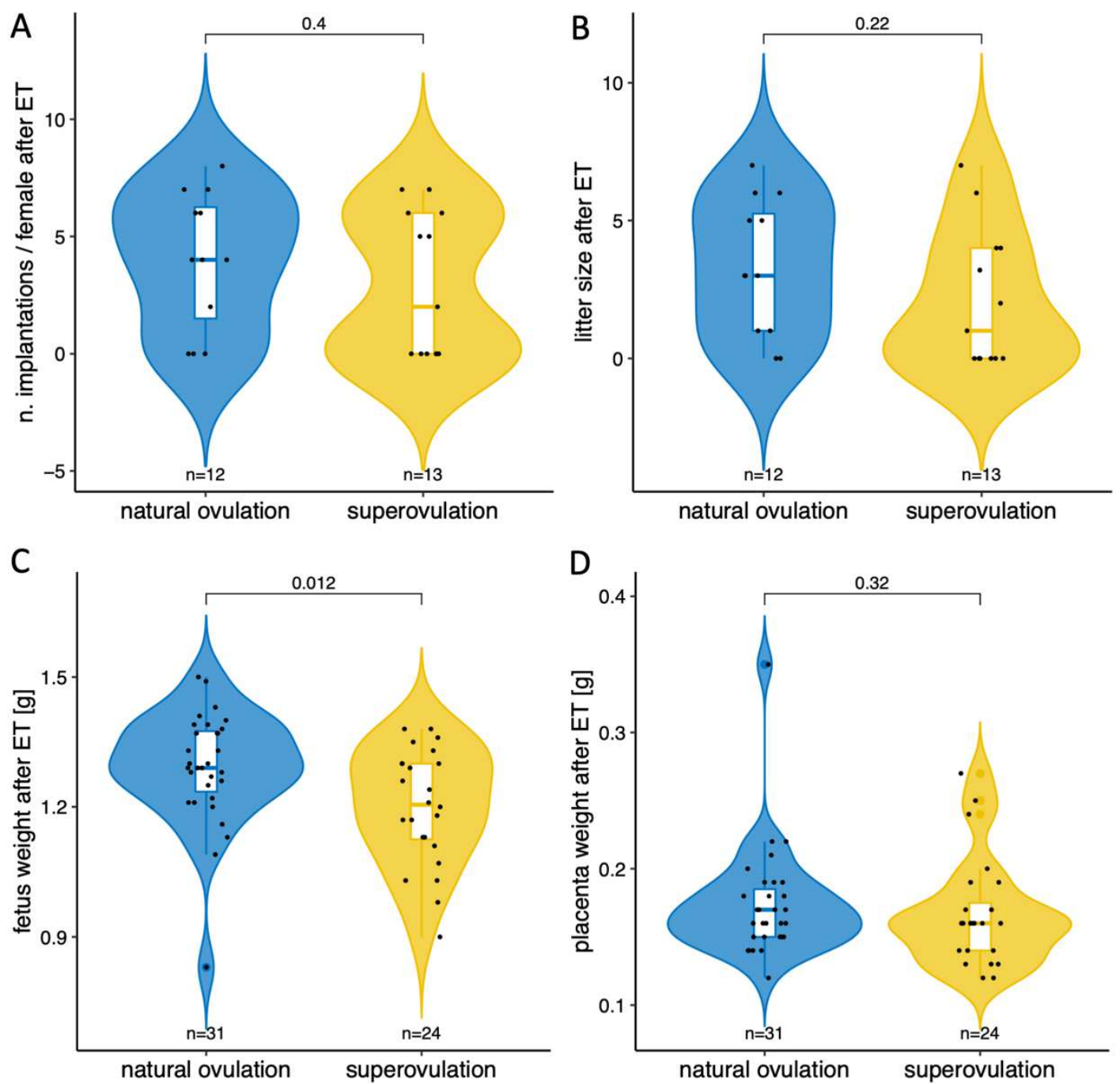

Figure 5. Postimplantation performance of superovulated and naturally ovulated oocytes. Embryo transfers were conducted with at least 10 recipients per group relying on fixed numbers of 4-cell stage embryos ( $n=8$ per pseudopregnant female). These were transplanted to the oviduct, ensuring that i) the oocytes used were competent for cleavage, ii) time in culture was not unnecessarily long, iii) the later fetal densities in the uteri were balanced between the two experimental groups, and iv) undefined cues emanating from a stimulated genital tract were removed. (A) Number of implantations and (B) number of fetuses recorded at hysterectomy, 18 days after the transfer of 8 embryos to each pseudopregnant female. Each data point corresponds to one embryo transfer i.e. one mother. $P$ values refer to differences of numbers of implanted embryos or number of fetuses between groups (Wilcoxon test). (C) Birth weight and (D) placental weights of the individual fetuses. Each data point corresponds to one fetus or pup. $\mathrm{P}$ values refer to differences of weight between groups (Wilcoxon test). Abbreviations: $n$, number of; $E T$, embryo transfer. 


\section{In search of mechanisms: evidence of an insufficient duration of ovarian oocyte maturation in the etiology of the adverse superovulation effects}

Two hypotheses are suited to explain the origin of the proteome perturbation and reduced developmental fitness after superovulation. The first hypothesis considers that the exogenous gonadotropins per se made the oocyte's proteome depart from the norm, in a direct fashion. The second hypothesis considers that the exogenous gonadotropins altered the oocyte's normal trajectory of maturation, and this indirectly influenced the oocyte composition. In both cases the proteome is expected to be different from that of naturally ovulated oocytes, but for different reasons. Albeit both hypotheses are not mutually exclusive, two lines of evidence favor the latter over the former.

As a first line of evidence, we are going to show that the more advanced oocytes (during oogenesis) have thicker zonae. Specifically, when oocytes were matured in vitro (IVM) starting from ovarian precursors that that were known a priori to have attained a higher vs. lower degree of maturation, resultant MII oocytes were distinguishable by ZP thickness and vitellus diameter. This difference underlines the importance of the time available for oocyte maturation. To determine the degree of maturation of the oocytes, we took advantage of the known relationship between extent of maturation and chromatin distribution in the oocyte's germinal vesicle ${ }^{58}$ : briefly, more advanced oocytes have a germinal vesicle with a surrounded nucleolus (SN), while less mature oocytes have a nonsurrounded nucleolus (NSN). Interestingly, upon IVM, the SN-derived MII oocytes narrowed the deficit of ZP thickness and cell diameter compared to NSN oocytes (Figure 6A,B). Since oocyte maturity and size are interdependent and they matter for development, we dissociated the two factors to facilitate interpretations. The sole reduction of ooplasm volume by $20 \%$ via aspiration did not exacerbate the preimplantation losses (blastocyst rate $80 \%, n=35 ; 28 / 35$; sham manipulation, 64\%, n=26; P-value $=0.24$, Fisher's exact test) nor the imbalance of blastocyst composition (Supplementary Figure S6). This observation suggests that superovulation brakes the development of the oocytes before they complete maturation, and leads us to the second line of evidence.

As a second line of evidence, we are going to show that a shorter vs. longer time in maturation results in a change of oocyte's proteome composition. Specifically, the 
proteomic differences between superovulated and naturally ovulated oocytes were remodeled when the time interval between the administration of the two gonadotropins was increased from $48 \mathrm{~h}$ to $72 \mathrm{~h}$. An important prerequisite for the $48-72 \mathrm{~h}$ extension was the knowledge that the half-life of eCG in vivo is up to 5-6 days ${ }^{59,60}$, which includes the $72 \mathrm{~h}$. The extension of the time interval did not have any evident consequences on the number of oocytes found in the oviduct after hCG $(26.9 \pm 10.3$ and $24.4 \pm 13.2$ after $48 \mathrm{~h}$ and $72 \mathrm{~h} ; \mathrm{n}=538$ and 414 , respectively; P-value $=0.53$, Wilcoxon test). However, the increase from $48 \mathrm{~h}$ to 72 $h$ resulted in a splitting of the superovulated proteomes (TMT) in two groups, as revealed by hierarchical clustering (Figure 7A) and PCA (Figure 7B; Supplementary Table S7). In particular, PCA separated the superovulated oocytes of $48 \mathrm{~h}$ and the superovulated oocytes of $72 \mathrm{~h}$ in the $3^{\text {rd }}$ component (PC3, 11\% of the variance, Figure $\left.7 C, D\right)$, which is the largest effect after that of superovulation compared to natural ovulation $\left(1^{\text {st }}\right.$ component, $P C 1, \sim 34 \%$ of the variance, Figure $7 \mathrm{~B}, \mathrm{C})$ and the effect of IVM ( $2^{\text {nd }}$ component, PC2, $\sim 21 \%$ of the variance, Figure $7 B, D)$.

With the comfort of these proteomic differences, we re-examined the morphological and functional parameters previously found to differ between superovulated and naturally ovulated oocytes. Morphologically, the diameters of the oocytes of the $72 \mathrm{~h}$ regime were closer to those of the natural ovulation group (median $78.8 \mu \mathrm{m}$ vs. $79.5 \mu \mathrm{m}$ ) than the diameters of the oocytes matured in $48 \mathrm{~h}$ (median $77.9 \mu \mathrm{m} ; \mathrm{p} \leq 0.028$ ), whereas no changes of ZP thickness could be reliably measured in the time from $48 \mathrm{~h}$ to $72 \mathrm{~h}$ (Figure 6A,B). During embryonic development in vitro, the embryos derived from the oocytes of the $72 \mathrm{~h}$ regime were almost never arrested prior to blastocyst stage, thereby resembling their natural ovulation counterparts ( $P$-value $=0.0013, \chi^{2}$ test; Figure $6 \mathrm{C}$ ). After embryo transfer to pseudopregnant recipients, the litter sizes of oocytes matured in $72 \mathrm{~h}$ were indistinguishable from those of the natural ovulation and were superior to those of the $48 \mathrm{~h}$ superovulation (Figure 6D). As the newborns were scored for overall normalcy, those of the $72 \mathrm{~h}$ regime did not present any obvious anomalies, and their body weight was similar to that of the natural ovulation group $(1.25 \pm 0.14 \mathrm{~g}$ vs. $1.29 \pm 0.13 \mathrm{~g} ; \mathrm{n}=48$ and 31 , respectively; $p=0.056)$. Hence, extending the ovarian residency appears to alleviate the quality problem posed by the superovulation. This consideration is consistent with the previous results, in which volume change alone proved insufficient to alter the developmental quality (Supplementary Figure S6). 
Collectively, the above evidence indicates that superovulated oocytes share characteristics with oocytes that are recruited for ovulation before their maturation is completed. This is consistent with these deficits appearing also in immature oocytes induced to mature in vitro, and with the correction of the deficits upon increase of the time between eCG and hCG.
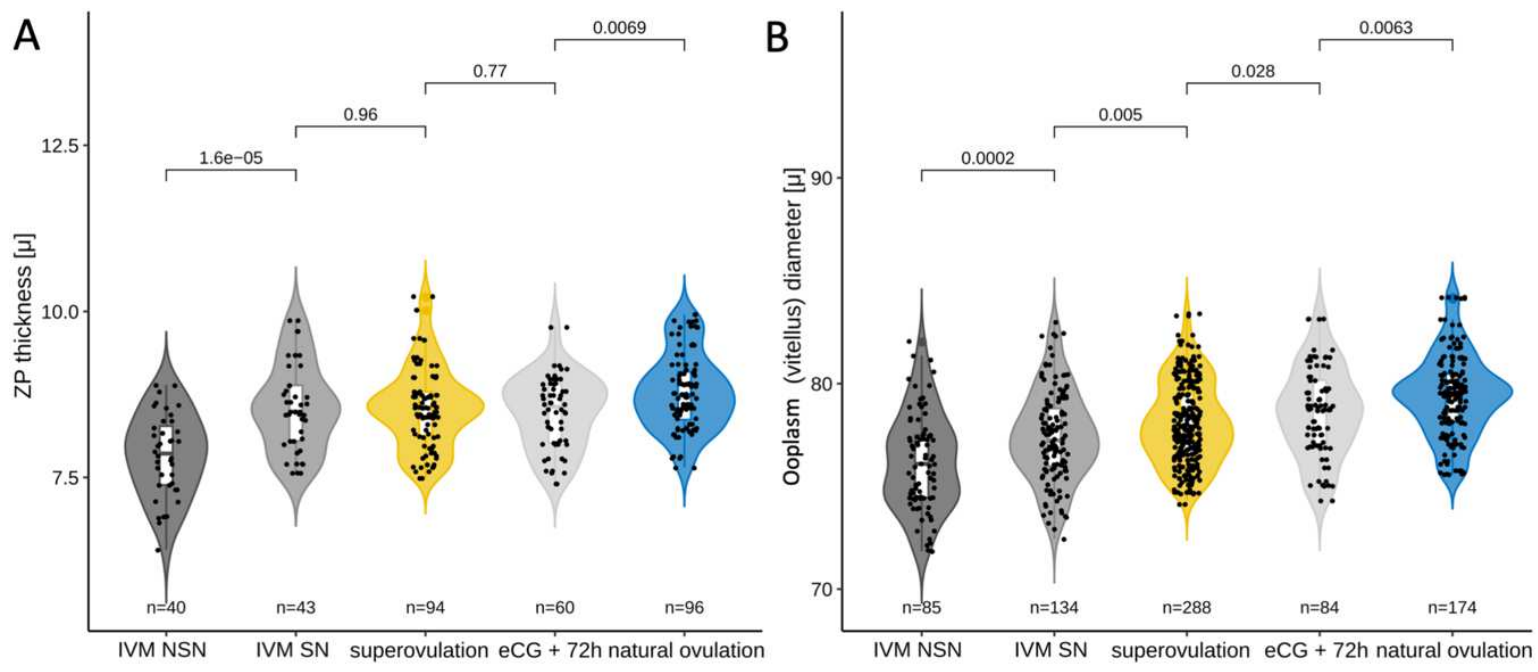

C
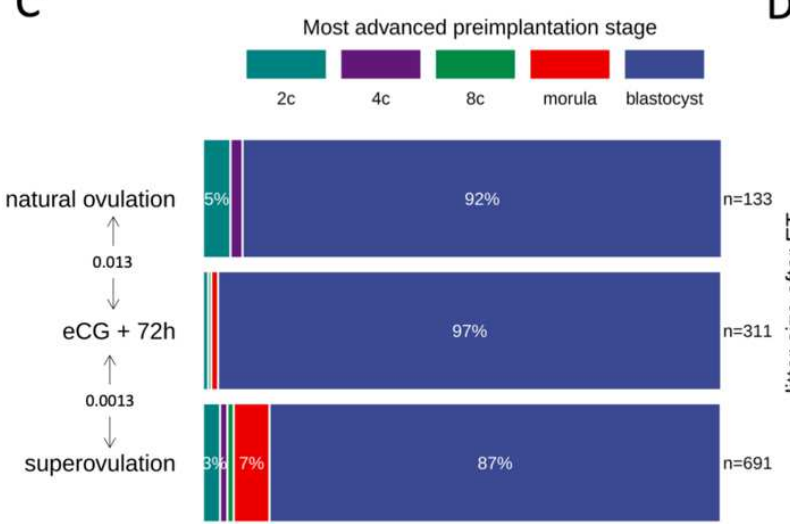

D

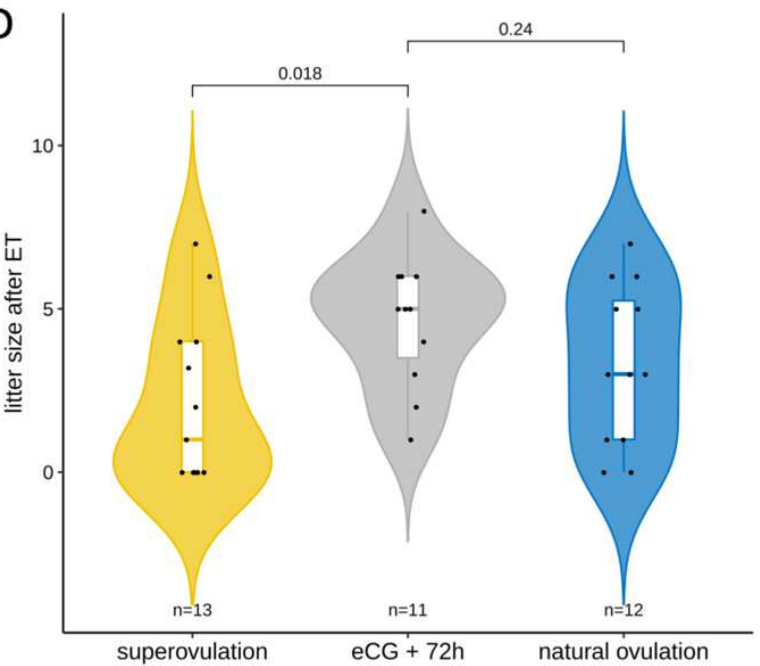

Figure 6. Effect of retrieving oocytes before or after the standard $48 \mathrm{~h}$ period of ovarian maturation. (A) The thickness of the ZP in MII oocytes derived from germinal vesicle oocytes prior to the ovulatory stimulus of hCG (IVM), compared to oocytes ovulated after the natural stimulus or after the exogenous stimulus ( $48 \mathrm{~h}$ vs $72 \mathrm{~h}$ ). Each data point corresponds to one oocyte. P-values refer to the differences of ZP thickness between groups (Wilcoxon test). (B) Diameter of oocyte proper (vitellus) in the same oocytes as described in (A). Each data point corresponds to one oocyte. P-values refer to differences of oocyte diameter between groups (Wilcoxon test). (C) The most advanced preimplantation stage reached in $\operatorname{KSOM(aa)~by~the~embryos~of~superovulated~}$ oocytes that spent $48 \mathrm{~h}$ vs $72 \mathrm{~h}$ in the ovary, compared to naturally ovulated oocytes cultured. Note that the scoring started from the 2-cell stage, since arrested 1-cells could be either oocytes which were not fertilized, or fertilized oocytes which failed to cleave. $\chi^{2}$ test. (D) Number of fetuses recorded at hysterectomy, 18 days after 
the transfer of 8 embryos per pseudopregnant female. Each data point corresponds to one embryo transfer or mother. P values refer to differences of litter size between groups (Wilcoxon test). Abbreviations: IVM, in vitro maturation; NSN, non-surrounded nucleolus; SN, surrounded nucleolus; eCG, equine chorionic gonadotropin; ZP, zona pellucida; ET, embryo transfer.

\section{A}

Proteome of MII oocytes
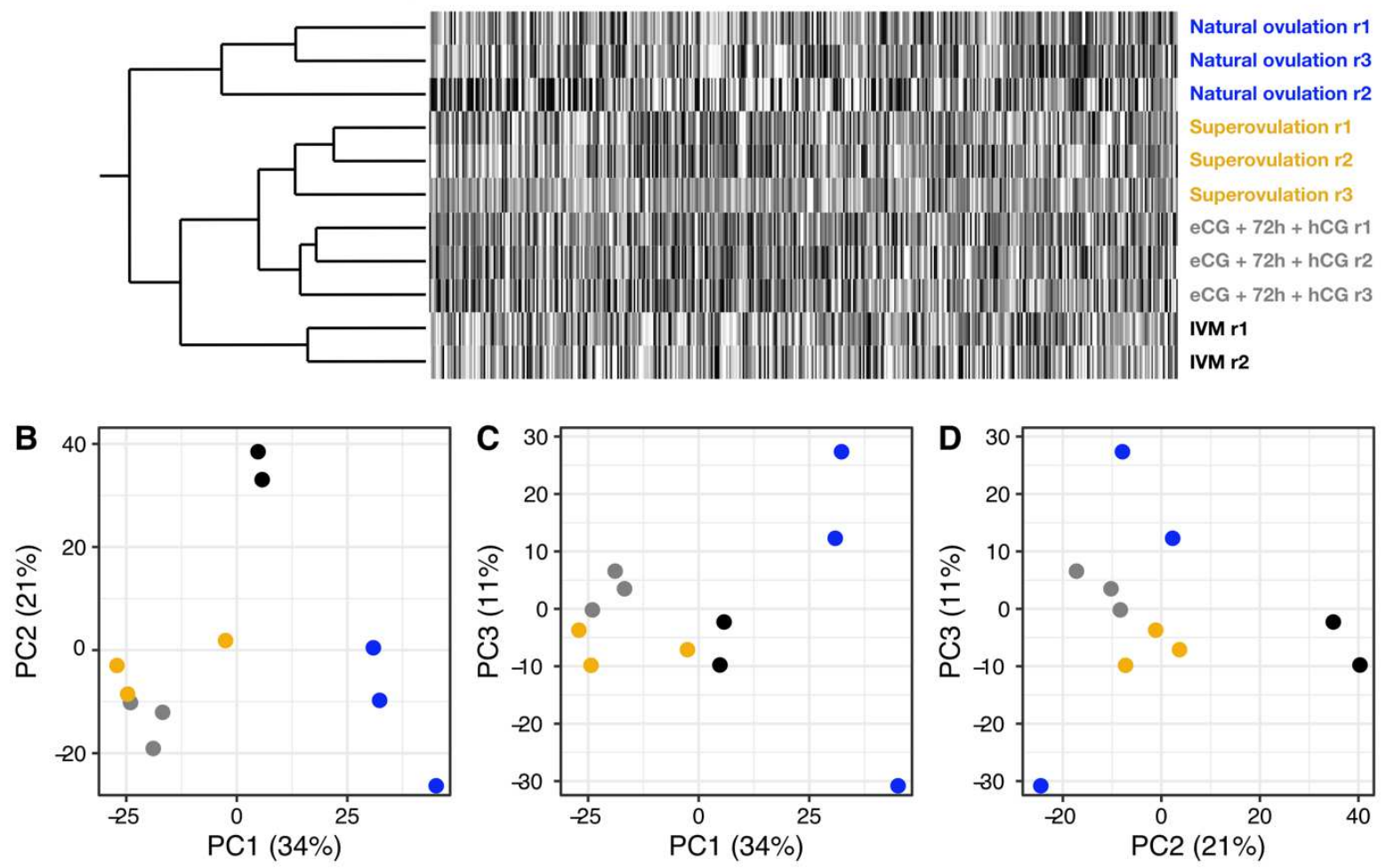

Figure 7. Proteome analysis (TMT) of oocytes superovulated with the $72 \mathrm{~h}$ regime compared to conventionally superovulated, naturally ovulated or IVM oocytes. Hierarchical clustering of the proteome $(A)$ and principal component analysis $(B, C, D)$. Each data point corresponds to one replicate. Oocytes superovulated with the $72 \mathrm{~h}$ regime form a separate subcluster $(A)$. The $1^{\text {st }}$ component of the PCA resolves the naturally ovulated oocytes from all the others $(B)$. The $2^{\text {nd }}$ component of the PCA resolves the IVM oocytes from all the others $(B, D)$. The $3^{\text {rd }}$ component of the PCA almost resolves the superovulated of the conventional stimulation protocol from the superovulated oocytes of the eCG $+72 h+h C G$ protocol (C,D). Samples were derived from pools of 200-250 oocytes, in triplicate for each treatment (except IVM, in duplicate). Abbreviations: IVM, in vitro maturation; eCG, equine chorionic gonadotropin; hCG, human chorionic gonadotropin. 


\section{Discussion}

It is commonly held that oocytes ripened via superovulation possess developmental abilities that are very similar to those obtained after natural ovulation, and that residual differences are due to unrelated, i.e., non-intrinsic causes. If it were not so, then superovulated oocytes would not be suited to derive conclusions of general validity about development, and human assisted reproduction might be less safe than we think. We hypothesized that proteomic data, missing to date, may provide new insights into the matter. As our study shows, while the transcriptomes of superovulated oocytes and derivative embryos are very similar to their naturally ovulated counterparts, the proteomes are clearly distinguishable. We are going to elaborate that the superovulation is not inflicting direct injury on the oocytes, but causes them to be released from the ovary prematurely, before their proteomes reach a state equivalent to that of natural ovulation. This proposal allows the reconciliation of the different views on superovulation: the view that superovulated oocytes are identical to naturally ovulated ones (with differences being due to confounding factors, for example), and the view that superovulated oocytes are inherently different from naturally ovulated ones (as documented for specific genes). Both views are correct, depending on the gene expression product, whether transcript or protein, and on the time elapsed until superovulation.

One consideration regarding the experimental design was fundamental in our study, namely: to compare the embryos from superovulated and naturally ovulated oocytes on an equal footing. This cannot be accomplished in vivo (although it is still the prevalent way ${ }^{38,51}$ ), but must be done in vitro and/or by transfer to an non-stimulated genital tract, because the superovulation exerts influences on the environment of the oviduct and uterus, which are passed on to the embryos (see Introduction). It was beyond the scope of this study to define these oviductal influences, which were therefore excluded from our experimental design, by culturing the fertilized oocytes in vitro and by testing their postimplantation potential in naturally cycling females (naturally mated to vasectomized males). Our in vitro-cultured superovulated and naturally ovulated oocytes were subjected to tandem transcriptome and proteome analysis of seven developmental stages up to blastocyst. Functional enrichment 
analysis of the differentially expressed proteins returned embryo-relevant ontology terms, such as "thin zona pellucida" (ZP), "embryonic growth arrest", and "absent inner cell mass proliferation", which take center stage in the first part of the discussion. How these phenotypic traits arose in superovulated oocytes and embryos, and why they were not discovered before, forms the second part of the discussion.

Although we were interested in comparing our genome-wide expression data to those in the literature, we could not find other proteome studies of mouse embryos derived from naturally ovulated oocytes, this being the first one. Surrogating the transcriptome for the proteome, the inspection of mRNA studies was problematic too, due to the paucity of studies and, in suborder, to the differences in methods and experimental designs. While superovulated oocytes have been analyzed for gene expression, e.g., transcriptome profiling, several times (reviewed in ${ }^{61}$ ), their natural counterparts were often missing, and the oocytes were seldom followed up during development. In one of only two transcriptomic studies eligible for comparison ${ }^{41,62}$, blastocysts were recovered from the stimulated genital tract and 92 genes were found perturbed ( 1.5 fold-change, $P$-value $<0.01)$, but it is not clear if these perturbations were caused by oocyte quality or by the environment of the stimulated genital tract ${ }^{41}$. In a second study, 117 of 11,110 genes were found differentially expressed between oocytes from eCG-primed and unprimed animals ${ }^{62}$, but the oocytes were at the germinal vesicle stage and thus were not directly relevant for reproduction ${ }^{62}$. Apart from these genome-wide studies, the effects of superovulation on mouse oocytes were studied on a small scale by candidate gene approaches. Using qPCR and/or immunofluorescence, one study reported a significant reduction of maternal effect gene expression (Bmp15, Hdgf, Dnmt1, Dppa3, and $\left.Z f p 57 ;{ }^{21}\right)$, another study reported partial reduction of the amount of DNA methyltransferase protein ${ }^{23}$, and a third study reported altered levels of Epab and Pabpc1 ${ }^{42}$. However, our datasets (this study) do not show effects on Bmp15, Hdgf, Dnmt1, Dppa3, Zfp57 and other gene products previously reported, suggesting they may be due to confounding influences by the genital tract.

A remarkable finding of our study was that the oocytes and embryos - which had been manually deprived of ZP - featured just the ZP proteins among the differentially expressed proteins of the superovulation vs. natural ovulation. We validated the precondition of this finding, namely presence of ZP inside the cell, by an independent method. When Western blot 
analysis was applied, the amount of Zp3 was nearly identical in the ZP-free and ZP-enclosed samples, thereby indicating that the bulk of Zp3 is intracellular. This observation is in line with the immunofluorescent detection of Zp3 in the mouse ooplasm ${ }^{52}$. It is known that the expression of ZP transcripts is high in growing mouse oocytes and decreases to undetectable levels after fertilization ${ }^{63,64}$; these properties justify the status of ZP genes as oocyte-specific. Given this background, how could the ZP proteins be differentially expressed in oocytes and derivative embryos which were deprived of the ZP? Previous reports have documented the presence of ZPs on the plasma membrane of mouse oocytes ${ }^{65-67}$. In the light of this information, we checked if the different ZP levels measured by proteomics were reflected in the thickness of the ZP. Indeed, we verified that the ZP was thicker in the naturally ovulated oocytes than in the superovulated oocytes. This is reminiscent of the variable ZP thickness reported in human oocytes after IVM, in which next to the ZP, also the oocyte proper (vitellus) was found to vary in size ${ }^{68,69}$. Therefore, we checked if the superovulated mouse oocytes with thinner or thicker ZP also presented different diameters, which was the case. Our finding contrasts with the prevalent view that superovulated and naturally ovulated oocytes have similar diameters. We deem that the difference has been overlooked until now due to limited sample sizes ${ }^{26}$, to reports made available only by personal communication (e.g. ${ }^{70}$ ) or to use of data obtained from non-conventional protocol of superovulation ${ }^{71}$. As of March $8^{\text {th }} 2021$, an ontology term related to 'small oocyte' has not been included in the Mammalian Phenotype Browser yet. Given our findings, we propose that a gene ontology category 'small oocyte' is worth considering, as companion of the term "regulation of cell size" (GO:0008361), which does not - for example - include the $Z p$ genes although it is established that secretion of the zona and extent of oocyte growth are interrelated.

Another remarkable finding of our study was that the superovulated oocytes also gave rise to embryos with more frequent - and later occurring - arrest at preblastocyst stages, and that these blastocysts contained an inner cell mass depleted of primitive endoderm and epiblast, supporting the MP terms "embryonic growth arrest" and "absent inner cell mass proliferation". One would expect that properly functioning embryos discard their unfit members as soon as possible, which is what naturally ovulated oocytes did, already at the 2-cell stage; in contrast, superovulated oocytes were selected later, at the morula stage. We cannot explain the mechanism underlying the delay, but we note the chronological proximity between the morula stage and the stage when the proteome is most 
perturbed (8-cell stage; this study), in accord with our previous work ${ }^{46}$. This chronological proximity supports that the later developmental arrest of superovulated oocytes was a consequence of a disturbance of protein expression. At the blastocyst stage, we recorded a depletion of the inner cell mass under the same gonadotropin dosage (10 I.U.) as used in another study ${ }^{14}$, although the mouse strains were different. The depletion of epiblast and primitive endoderm cells echoes our previous work ${ }^{72}$, in which we reported on a small - but not negligible - proportion of non-manipulated, in vivo-produced blastocysts lacking epiblast cells in their inner cell masses. This phenotype is consistent with relevant proteins returned by our GO analysis, namely Dab2 and Cops5 (Supplementary Table S6), which are bona fide markers of the primitive endoderm and epiblast sub-compartment of the inner cell mass and whose genetic mutants feature defects of cell proliferation ${ }^{73-78}$. In contrast to the inner cell mass and its sub-compartments, the trophectoderm cell numbers were the same in the two groups, in line with a report that embryo differentiation, particularly in trophectoderm cells, is influenced by embryo culture (identical for the two groups in our study) rather than by ovarian stimulation ${ }^{51}$.

A key issue pertains to the origin of the smaller oocytes with reduced embryonic fitness after superovulation. An obvious question is whether they had reduced fitness because they were smaller, or whether the smaller size was an epiphenomenon. We thus tested if making the oocyte volume even smaller $(-20 \%)$ would exacerbate the problem. The volumereduced oocytes formed blastocysts at the same rate as sham-manipulated controls, and their germ layer composition was unaffected. Even though a $20 \%$ reduction cannot possibly change protein abundances by e.g. the $>2$-fold encountered in this study (in case of superovulation), we deem it safe enough to conclude that reduced volume is a mere epiphenomenon, which does not per se cause the inferiority of superovulated oocytes. Instead we propose a model whereby the superovulation treatment stops the oocytes prematurely on their way to attain the proper size and natural quality. A similar proposal has been made in an independent study, on the basis of DNA methylation data in superovulated vs. naturally ovulated mouse oocytes ${ }^{27}$.

If the above model is correct, then the logical follow-up experiment is to allow for more time until the release of the oocytes from the ovary, expecting to see a relief of the superovulation-associated deficits. Unlike the gonadotropin dosage $27,79,80$ and the time of 
gonadotropin stimulation in relation to estrous cycle ${ }^{81}$ and photoperiod ${ }^{82}$, the interval of the gonadotropins has not received much consideration, although it goes by itself that the accumulation of gene products in oocytes is a function of time during oogenesis. Compared to the natural length of the ovarian cycle in F1 mice, which is 4 days ${ }^{83}$, the interval eCG-hCG of the conventional superovulation spans only 2 days, thereby triggering the idea to assess the extent of preovulatory maturation attained until the administration of hCG. While a longer eCG-hCG interval had been tested before, it reached up to $60 \mathrm{~h}$ with conflicting results ${ }^{39,40}$. We thus increased the interval between eCG and hCG to $72 \mathrm{~h}$ - time that still supports ovulation in Calomys ${ }^{84}$ and in mice ${ }^{85}$ without incurring preovulatory aging ${ }^{86}$. In global terms of proteome composition, the oocytes released from the ovary after $72 \mathrm{~h}$ were distinguishable from the conventionally superovulated ovulated oocytes. However, the differences were small at the level of the individual proteins. This led us to consider that oocyte quality is unlikely the outcome of expression of a restricted number of genes; rather, it is the outcome of a broad gene network, which poses a demand for molecular e.g. proteomic approaches possibly even more refined and sensitive than the approach we used here. The oocytes released from the ovary after $72 \mathrm{~h}$ had a diameter which was closer to that of naturally ovulated oocytes. The similarity of the two groups emerged also from the occurrence of preblastocyst arrest, which almost never occurred in the $72 \mathrm{~h}$ protocol. Postimplantation development also seemed to benefit from the extension, whereby the litter size after $72 \mathrm{~h}$ was no longer inferior to that of the natural ovulation. These improvements after $72 \mathrm{~h}$ support that the oocytes had stopped prematurely on their way to attain the proper size and quality in the conventional superovulation regime. It may be noted that a longer stimulation can be beneficial also in human reproduction ${ }^{87}$.

Unlike the three preimplantation terms examined above ("thin zona pellucida", "embryonic growth arrest", and "absent inner cell mass proliferation"), the ability to implant in naturally cycling females was not significantly different, and we did not detect any obvious phenotypic differences between fetuses of superovulated and naturally ovulated oocytes after embryo transfer, except for a slight reduction of body weight in the superovulation group. This reduction is consistent with independent mouse study ${ }^{37}$ and it may be noted that also human oocytes with a background of hormonal stimulation gave rise to babies with reduced birth weight, and this reduction was observed no matter if the uterus had been stimulated or not ${ }^{88,89}$. Thus, the lighter body weight is rooted primarily in the oocytes. The 
lack of major fetal anomalies is in line with our previous study ${ }^{90}$ in which we reported that preimplantation effects of embryo culture media do not persist after implantation. A critique may be voiced that the present study was not sufficiently powered to measure postimplantation and postnatal effects of the treatment. Although it was proposed that the number of experimental animals could be increased until statistical significance is reached ${ }^{91}$, this is not an ethically acceptable solution in the face of the efforts to reduce the numbers of animals used in science. As a matter of fact, the 12-13 recipients per group in our study are a respectable number, in comparison to other similar studies $29,30,33,34,37,38$. We also acknowledge the fact that there may be other, more subtle or long-term differences between superovulated and naturally ovulated oocytes, than the ones we found at preimplantation. Even with these limitations, we deem we can state confidently that problems associated with superovulation in mice are not as pronounced at postimplantation as they are at preimplantation. This statement seems to be at variance with the conclusion of an independent study reporting that "preimplantation embryo exposure to superovulation affects placental growth, whereas peri-implantation exposure affects fetal growth" ${ }^{38}$. Both growths were unaffected in our study. One experimental difference between the two studies is that zygotic cleavage took place in vitro in our study, whereas in the other study blastocysts were flushed from the uterus ${ }^{38}$. Together, the pre- and postimplantation data of our study converge on a scenario of negative selection of the unfit or unviable embryos as occurring during preimplantation, whereby there remains less, if anything, left for later selection.

In summary, our tandem transcriptome and proteome analysis allowed the reconciliation of the different views on the similarities and differences between superovulated and naturally ovulated oocytes: they are similar on the transcriptome level, but different on the proteome level. Since the latter is closer to phenotype, we advocate that global gene expression studies to come should take the protein dimension into account, since this information cannot be reliably inferred from transcriptome studies. Striking was the example of the ZP, which is abundantly present inside the denuded oocyte (in spite of its role as extracellular coat) and continued to be detected in the embryos up to blastocyst (in spite of undetectable transcript levels). Perhaps if we started to look even deeper into the proteomes, we might discover many other cases of gene products with hitherto unsuspected functions. Again, the ZPs offer a candidate to explain unsuspected functions on the molecular level, given the ZP interaction partners (e.g. Ptprk, Aipl1, Diaph2) suggested to operate in the cell 
nucleus ${ }^{52}$, in the face of the extracellular role ascribed to the ZPs so far. If we continue to rely on transcripts in lieu of transcripts plus proteins, our molecular understanding of oocyte quality will stay woefully incomplete. If we persist in using the routine $48 \mathrm{~h}$ protocol which prevents superovulated oocytes to attain their proper size, oocyte quality itself may stay lower than necessary. An evidence-based protocol for superovulation may be quite different from the one currently used routinely in mice. 


\section{Materials and Methods}

Compliance with regulations on research animals. All mice used were maintained in individually ventilated type $2 \mathrm{~L}$ cages in the animal facility of the MPI Münster, with a controlled temperature of $22 \circ \mathrm{C}$, a 14/10 h light/dark photoperiod (light on at 6:00 a.m.) and free access to water and food (Teklad 2020SX, Envigo). Procedures used in this study followed the ethical guidelines of the European Laboratory Animal Science Associations (FELASA) and the ARRIVE reporting guidelines ${ }^{92,93}$. On the local regulatory level, mice were used for experiments according to the ethical approval issued by the Landesamt für Natur, Umwelt und Verbraucherschutz (LANUV) of the state of North RhineWestphalia, Germany (Permit number 84-02.04.2016.A229; 81-02.04.2017.A432).

Gonadotropin stimulation regime. Equine chorionic gonadotropin (eCG), also known as pregnant mare serum gonadotropin (PMSG) and human chorionic gonadotropin (hCG) were obtained from Intervet as commercial products Intergonan and Ovogest, respectively (MSD Tiergesundheit, Germany). Lean B6C3F1 mice aged 8-to-10 weeks were injected intraperitoneally, using a $27 \mathrm{G}$ needle, at 5 pm, with 10 I.U. eCG and 10 I.U. hCG 48 hours apart, as per the conventional superovulation protocol in use since $1957^{4}$. Ten I.U. correspond to $128 \mathrm{ng}$ eCG ${ }^{60}$ and compare to the $335 \mathrm{ng} / \mathrm{mL}$ FSH found in the plasma of $\mathrm{C} 57 \mathrm{Bl} / 6$ mice ${ }^{94}$. In one set of experiments, the interval between eCG and hCG was increased to 72 hours.

\section{Collection and morphometry of unfertilized and fertilized oocytes from natural and}

stimulated cycles. For natural ovulation, eight- to ten-week-old B6C3F1 females were injected with sole vehicle used to dissolve the gonadotropins (PBS) and caged three days with vasectomized or stud CD1 males (6-12 months old). A vaginal plug was taken as indicating that mating had occurred. On the third day after caging, at 8 a.m., metaphase II (MII) oocytes or fertilized oocytes (1-cell embryos) were isolated from those females that received a copulation plug from the vasectomized or stud males, respectively, on that day. Females were killed by cervical dislocation. For superovulation, B6C3F1 females were injected with eCG and hCG 48 or 72 hours apart at 5 p.m., and upon the second injection were caged one night with vasectomized or with stud CD1 males. On the following day at 8 
a.m., plugged females were killed and MII oocytes or fertilized oocytes were isolated. The room temperature of oocyte isolation and handling was $28 \circ \mathrm{C}$. Cumulus-oocyte complexes were collected by tearing the oviducts 15 hours after hCG injection and released into Hepesbuffered Chatot, Ziomek and Bavister medium (HCZB) ${ }^{95}$ with bovine serum albumin (BSA) replaced by polyvinylpyrrolidone $(40 \mathrm{kDa})$. Cumulus cells were removed in hyaluronidase (cat. no. 151271, ICN Biomedicals, USA; 50 I.U./mL in HCZB) on the stage of a stereomicroscope fitted with camera. We were very careful at measuring the oocyte proper (vitellus) rapidly and synchronously for the two groups, being aware that oocyte volume is not a constant, but it is dynamically regulated ${ }^{96-98}$. The time between initiation of the hyaluronidase treatment and imaging did not exceed 20 minutes. Images were processed using NIH ImageJ software, manually drawing a line across the zona pellucida (ZP) perpendicular to its surface, and measuring the zona thickness directly; the oocyte perimeter was drawn along the oolemma with the free-hand tool. The MII oocytes from natural ovulation and superovulation were transferred to fresh HCZB on the stage of an inverted microscope, and imaged side by side using a 10X objective. Measures were converted from pixels to $\mu \mathrm{m}$ with a calibrated micrometer slide. The oocyte diameter was obtained by dividing the perimeter by pi ( 3.141).

In vitro production of MIl oocytes from germinal vesicle oocytes. To obtain MII oocytes from germinal vesicle-stage oocytes via in vitro maturation (IVM), germinal vesicle-stage oocytes were retrieved from the ovary $48 \mathrm{~h}$ after eCG by puncturing its surface in HCZB. Oocytes were deprived of surrounding cumulus cells by gentle pipetting and incubated for 20 minutes at $37 \circ \mathrm{C}$ in $\mathrm{HCZB}$ added with Hoechst $0.05 \mu \mathrm{g} / \mathrm{mL}$. Fluorescent imaging was performed on the stage of an inverted microscope using a 20X S-Fluor objective. The oocytes were sorted into two groups according to the distribution of chromatin around the nucleolus (a surrounded nucleolus, SN, or a non-surrounded nucleolus, NSN; ${ }^{58}$ ) and allowed to mature overnight in $\alpha$-MEM (cat. no. M4526, Sigma-Aldrich Chemie GmbH, Germany) supplemented with $10 \%$ fetal bovine serum and 50 I.U./mL gentamicin sulfate (MP Biomedicals). The SNMII and NSN-MII oocytes were imaged side by side using a 10X objective, as described for the (super)ovulated oocytes.

In vitro embryo culture for proteome, transcriptome and phenotypic analysis. MII or fertilized oocytes were transferred to $500 \mu \mathrm{L}$ of potassium (K) simplex optimization medium 
enriched with aminoacids (KSOM(aa)) in a 4-well plate without oil overlay, at $37 \circ \mathrm{C}$ under $6 \% \mathrm{CO}_{2}$ in air. $\mathrm{KSOM}(\mathrm{aa})$ was synthesized in house from individual components and included $0.5 \mathrm{X}$ EAA, $0.5 \mathrm{X}$ NEAA and $0.5 \mathrm{X}$ glutamine according to recipe ${ }^{99}$. KSOM(aa) was also added with $0.2 \%(w / v)$ BSA (Probumin, Serologicals Corporation, Celliance) and 50 I.U./mL gentamicin sulfate (MP Biomedicals). Developmental stages were collected from the plate at the desired time points (MII oocyte, $15 \mathrm{~h}$ post hCG (hphCG); 1-cell stage, $15 \mathrm{hphCG}$; 2-cell stage, 43 hphCG; 4-cell stage: 53 hphCG; 8-cell stage, 62 hphCG; morula: 72 hphCG; blastocyst, 92 hphCG; the non-stimulated counterparts were collected at the same time as the superovulated oocytes/embryos). For proteome analysis, oocytes and embryos were deprived of the ZP by pipetting in warm acidic Tyrode solution (cat. no. T1788, Sigma-Aldrich Chemie $\mathrm{GmbH}$, Germany) for 30-60 s and then rinsed in protein-free Hepes-buffered CZB medium (BSA replaced through polyvinylpyrrolidone $40 \mathrm{kDa}$ ). To produce each individual sample 200-250 oocytes or embryos were lysed in 15-20 $\mu$ l of sodium dodecyl sulfate (SDS) lysis buffer (4\% SDS, $50 \mathrm{mM}$ HEPES pH 7.5) and stored at $-80^{\circ} \mathrm{C}$ until further processing. For transcriptome analysis, an average of 210 oocytes or embryos per sample were lysed, still encased in their zona, in lysis buffer (Zymo Research, cat.no. R1051) and stored at $-80^{\circ} \mathrm{C}$ until further processing. The developmental stages were also inspected for the occurrence of cleavage arrest, and were sampled for embryo transfers and for the assessment of blastocyst's cell lineage allocation.

Proteome analysis of MII oocytes, pronuclear oocytes and preimplantation embryos. The following methods essentially follow those of our previous work ${ }^{45}$. To compare the developmental series between superovulation and natural ovulation we made use of our established analysis pipeline ( ${ }^{50}$, PXD003093; ${ }^{49}$, PXD000512) which is based on the 'stable isotope labeling by/with amino acids in cell culture' (SILAC) technique ${ }^{100}$. In addition, we used a multiplexing pipeline with tandem mass tag (TMT, ${ }^{101}$ ) to compare the sole MII oocytes between superovulation and natural ovulation.

For SILAC, samples were spiked with a standard prepared from F9 embryonal carcinoma (EC) cells ${ }^{102,103}$. F9 EC cells build tumors (teratomata) that are considered as caricatures of embryogenesis, because they can differentiate into almost every tissue ${ }^{104}$, therefore F9 EC cells afford an ample coverage of the proteins expressed in early embryos. Each oocyte or embryo lysate was supplemented with an equal amount of protein lysate from isotopically 
labeled (Lys8 and Arg10) F9 EC cells as SILAC spike-in standard (> 98\% labeling efficiency).

These 1:1 mixtures were then digested with Lysyl endopeptidase and trypsin, desalted, and fractionated by offline high-pH reversed-phase chromatography. Fractionation was accomplished in a concatenated fashion. Lastly, all samples were analyzed by liquid chromatography-mass spectrometry (LC-MS/MS) using a QExactive or QExactive HF mass spectrometer as described in our previous work. Data were obtained in duplicate, and raw data were processed for identification and quantification by MaxQuant Software (version 1.6.4.0, ${ }^{105}$ ) with the 'iBAQ' option enabled and the option 'requantify' disabled. For identification, the search was performed against the UniProt mouse database (release identifier UP000000589_10090.fasta) concatenated with reversed sequence versions of all entries and supplemented with common lab contaminants. Parameters defined for the search were trypsin as digesting enzyme, allowing two missed cleavages, a minimum length of 6 amino acids, carbamidomethylation at cysteine residues as fixed modification, and oxidation at methionine, and protein $\mathrm{N}$-terminal acetylation as variable modifications. The maximum allowed mass deviation was 20 ppm for the MS and 0.5 Da for the MS/ MS scans. Protein groups were regarded as identified with a false discovery rate (FDR) set to $1 \%$ for all peptide and protein identifications; in addition, at least two matching peptides were required and at least one of these peptides had to be unique to the protein group. We focused on the iBAQ values of the 'light' peptide versions only (= peptides derived from oocyte proteins but not from the F9 spike-in standard). The iBAQ algorithm allows to calculate the abundance of proteins within one sample by summing all peptide peak intensities detected for a given protein and normalizing it by the number of theoretically observable tryptic peptides for this protein. Thus, a mass-related measure (intensity) is transformed into a measure that is proportional to molar amounts (iBAQ). iBAQ values for each protein were then divided by the sum of all the $n$ iBAQ values for a given experiment to determine the molar fractional content of each protein $P($ riBAQ $)$ in a sample according to Shin et al. ${ }^{106}$, as follows:

$$
\text { riBAQ }=\frac{{ }_{B B A Q_{i}}}{\sum_{i=1}^{n}{ }_{B B A Q}}
$$


We applied nonparametric normalization, which is robust to outliers and anomalies in data that were generated in distinct LC-MS/MS runs. Specifically, proteins with riBAQp values greater than zero in fewer than two samples were discarded from further analysis. Remaining riBAQp values were averaged across replicates (if the protein had been detected in both replicates; otherwise, we used the single detected value), quantile-normalized, and $\log _{10}$ transformed. These values are further referred to as "expression values" of the proteins.

For TMT aimed at comparing the sole MII oocytes, we performed an 11-plex isobaric labelling experiment (isobaric reagents cat. no. 90110 and cat.no. A37724, Thermo Fisher Scientific, Germany) involving oocyte pools obtained by natural ovulation ( $n=803$ ), superovulation with conventional 48 h interval between eCG and hCG $(n=822)$, and superovulation with interval between eCG and hCG increased to 72 hours $(n=666)$. Each of the three pools of MII oocytes was divided equally into three technical replicates. We also included single samples of IVM SN oocytes $(n=351)$ as well as IVM NSN oocytes $(N=244)$, which served as outgroup samples to control for similarities/ dissimilarities in any subsequent dimensionality reduction analysis such as PCA. All samples were predigested with $0.5 \mu$ g endoproteinase LysC for $2 \mathrm{~h}$ at $37^{\circ} \mathrm{C}$, followed by dilution with $50 \mathrm{mM}$ ammonium bicarbonate buffer to lower the guanidinium hydrochloride concentration and a continuation of the digest over night after the addition of $0.5 \mu \mathrm{g}$ trypsin. Digestion mixtures were quenched by the addition of trifluoroacetic acid to a final concentration of $1 \%$ and desalted using C18 Stage tips. After drying samples in an Eppendorf Concentrator, peptides were resuspended in $20 \mu \mathrm{L} 200 \mathrm{mM}$ ethylpiperazinepropanesulfonic acid and TMT labelled using $5 \mu$ l of each labeling reagent (about $100 \mu \mathrm{g}$ ). Subsequent to mixing at equal ratios TMTlabeled samples were offline fractionated by high pH reversed phase HPLC on a YMC C18 column (2.1 x 100 mm; Buffer A 10 mM NH${ }_{4} \mathrm{OH}$; Buffer B 10 mM NH${ }_{4} \mathrm{OH} 90 \%$ acetonitrile; linear gradient from $3-40 \%$ B in $53 \mathrm{~min}$, flow rate $0.3 \mathrm{ml} / \mathrm{min}$ ). 15 concatenated fractions were collected, lyophilized and subjected to LC-MSMS on a Q Exactive HF instrument using a top 15 data dependent method (scan range $350-1600$ m/z; MS1 resolution 120,000; AGC target 3e6; maximum IT 50 ms; MS2 resolution 60,000; AGC target 1e5; maximum IT 108 ms; $\mathrm{NCE}=32 \mathrm{~V}$; dynamic exclusion enabled for $30 \mathrm{sec}$ ). Raw data were processed for identification and MS2-based quantification of reporter ions by MaxQuant Software (version 1.6.17.0, ${ }^{105}$ ). For identification, the search was performed against the UniProt mouse 
database (UP000000589_10090.fasta; release 04/2019) concatenated with reversed sequence versions of all entries and supplemented with common contaminants. Parameters defined for the search were trypsin as digesting enzyme, allowing two missed cleavages, a minimum length of 6 amino acids, carbamidomethylation at cysteine residues as fixed modification, and oxidation at methionine, and protein $\mathrm{N}$-terminal acetylation as variable modifications. The maximum allowed mass deviation was $20 \mathrm{ppm}$ for the MS and 0.5 Da for the MS/ MS scans. Protein groups were regarded as identified with a false discovery rate (FDR) set to $1 \%$ for all peptide and protein identifications; in addition, at least two matching peptides were required and at least one of these peptides had to be unique to the protein group.

Western blot confirmation of Zp3 protein as abundantly present in zona-free oocytes. The following methods essentially follow those of our previous work ${ }^{114}$. Zona-free oocytes were centrifuged in protein-free HCZB medium at $700 \mathrm{rpm}$ for $20 \mathrm{~min}$ to form a tiny pellet. The supernatant was carefully aspirated using a mouth-operated micropipette and replaced by RIPA buffer containing protease inhibitors. The resultant lysates were mixed with $6 x$ Laemmli sample buffer and boiled for $5 \mathrm{~min}$ at $99{ }^{\circ} \mathrm{C}$. These samples were loaded on a $12 \%$ polyacrylamide separation gel blotted onto a PVDF membrane. The membrane was blocked for at least $3 \mathrm{~h}$ and incubated (3\% nonfat dry milk in $0.1 \%$ PBS-Tween) with rabbit anti-ZP3 primary antibody (cat.no. PA5-89033, Thermo Fisher Scientific, Germany) applied at a dilution factor of 1:20000, overnight at $4{ }^{\circ} \mathrm{C}$. After $3 \mathrm{X}$ washing in $0.1 \%$ PBS-Tween, the blot was incubated with horseradish peroxidase-coupled secondary antibody at RT for $1 \mathrm{~h}$. The membrane was washed and then developed with chemiluminescent horseradish peroxidase substrate solution. The chemiluminescent signal was detected using the AGFA Curix 60. Signal intensities were standardized on $\alpha$-Tubulin whose antibody (cat. no. T6199, SigmaAldrich Chemie $\mathrm{GmbH}$, Germany) was applied at a dilution factor of 1:5000.

\section{Transcriptome analysis of MII oocytes, pronuclear oocytes and preimplantation embryos.}

Total RNA was converted to cDNA using the Smarter system (Takara) and sequencing libraries were prepared using the Nextera kit (Illumina). Libraries were sequenced on Illumina HiSeq3000 platform to obtain 43 million 36-base-single-end reads per library. Raw sequencing reads were trimmed, mapped to the Mus musculus Ensembl GRCm38 assembly, and quantified as previously described ${ }^{46}$. Only Ensembl gene identifiers for protein-coding 
genes associated with one or more reads were considered for further analysis. A matrix containing the number of reads mapped to each of the remaining Ensembl gene identifiers for each sample was used as input for the DESeq2 R/Bioconductor package (version 1.24.0, ${ }^{107,108}$ ) and normalized for library size with its default method. Regularized log-transformed values were obtained from the normalized counts, by applying DESeq2's rlogTransformation function with the "blind" parameter set to "TRUE" (i.e., free of experimental design). Regularized log-transformed values for Ensembl gene identifiers corresponding to the same gene symbol were averaged, and the values associated with each gene symbol were averaged across replicates. The resulting values are further referred to as "expression values" of the transcripts.

Differential gene expression analysis. Expression values for each protein/transcript were compared between the superovulation and natural ovulation groups using a two-sided exact permutation test based on the Wilcoxon signed-rank test statistic. The Wilcoxon signed-rank test is a non-parametric test used to assess differences in the mean ranks of two paired samples. Expression values were paired by stage. Thus, for each protein/transcript, the seven developmental stages of the oocyte and derivative embryos in the superovulation group were compared to their natural ovulation counterparts, obtaining one P-value. The resulting P-values were corrected with Benjamini-Hochberg's method (false discovery rate < $0.1)$.

Functional enrichment analysis. Functional enrichment analysis was performed with Enrichr 109,110 at https://maayanlab.cloud/Enrichr/. We selected the ontology 'Mammalian Phenotype' (MP), which builds on the Mouse Genome Informatics database and is therefore well suited to examine genes relevant to the mouse and its developmental biology. Terms with a FDR $\leq 0.01$ were considered enriched.

Data accessibility. The mass spectrometry proteomics data generated and analyzed in this study have been deposited to the ProteomeXchange Consortium via the PRIDE partner repository ${ }^{111,112}$ with the dataset identifier PXD021331 (SILAC) and PXD026347 (TMT). The RNAseq data analyzed in this study have been deposited to the DNA Databank of Japan Sequence Read Archive with the dataset identifier DRA005956 and DRA006335. A microarray dataset obtained from the same cellular material as the RNAseq has been 
deposited in the NCBI's Gene Expression Omnibus and is accessible through GEO Series accession number GSE110599.

For convenience, easy-access data tables of PXD021331, DRA005956-DRA006335, and PXD026347 are provided in supplementary material, as Supplementary Tables S1, S2 and S7, respectively.

Embryo transfer and post-implantation development. At the 4-cell stage, groups of 8 embryos were transferred surgically to one oviduct of pseudopregnant CD1 recipients. Pseudopregnancy was induced by mating untreated females with vasectomized CD1 males. On the day of the copulation plug the females weighed between 27 and $33 \mathrm{~g}$ and were older than 8 weeks but no older than 3 months. Prior to surgery, CD1 recipients were anesthetized with Ketamin (80 mg/kg body weight)/Xylazin (16 mg/kg)/Tramadol (15 mg/kg) in PBS, delivered intraperitoneally. The eyeballs were covered with eye ointment (Bepanthen) to protect the cornea. A 3-mm paralumbar incision was made in the peritoneum, the ovary and ancillary tissues were gently pulled out, and kept in position using Serafin clips. Drops of epinephrine solution $(0.1 \mathrm{mg} / \mathrm{ml}$ in PBS) were applied to the surface of the bursa ovarica to prevent possible bleeding when tearing the bursa to expose the oviduct and its infundibulum. Embryos were deposited in the infundibulum using a mouth-operated glass pipette with flame-polished tip. The ovary and ancillary tissues were returned to the original position and the wound in the skin was closed with metal clips. The surgery per se took typically $10-15$ min per mouse. The post-surgery recovery area was warmed to $\approx 30 \circ \mathrm{C}$ using infrared lamps. Animals were returned to their cages when fully awake. CD1 females were killed by cervical dislocation 18 days post-embryo transfer at E18.5, and then subjected to hysterectomy. The number of uterine implantation sites and resorption sites were counted for each animal. Fetuses and placentae were weighed and inspected for signs of obvious growth retardation and obvious external malformations, if any.

Experimental volume reduction of the ooplasm. Pronuclear-stage oocytes were incubated in $5 \mu \mathrm{M}$ Latrunculin B (dilution of 1000X stock in DMSO; cat. no. 428020, Merck Millipore, Darmstadt, Germany) diluted in Hepes-buffered CZB. Approx. 30-35 picoliters ooplasm were aspirated using a TransferTip (ES) pipette (cat. no. 5195000079, Eppendorf) operated by a CellTram Vario (Eppendorf). Manipulated oocytes were washed and returned to culture in KSOM(aa) for further development. 
Analysis of cell lineage allocation of blastocysts. Blastocysts from superovulated or naturally ovulated oocytes were analyzed by performing an immunostaining followed by confocal microscopy imaging to identify and map the different cell lineages, as described ${ }^{72}$, ${ }^{113}$. The following primary antibodies were applied simultaneously to the specimens overnight at $4 \circ \mathrm{C}$ : anti-Cdx2 mouse IgG1k (cat. no. CDX2-88, Emergo Europe, The Hague, The Netherlands), anti-Nanog rabbit IgG (cat. no. REC-RCAB0002P-F, Cosmo Bio, Tokyo, Japan) and anti-Sox17 goat IgG (cat no. AF1924, R\&D Systems) in dilutions of 1:200, 1:2000 and 1:100, respectively. Appropriate Alexa Fluor-tagged secondary antibodies (Invitrogen) were matched to the primaries and incubated for $2 \mathrm{~h}$ at room temperature. Embryos were placed in $5 \mu \mathrm{L}$ drops of PBS on a 50-mm thin-bottomed plastic dish (Greiner Bio-One, Lumox hydrophilic dish; Frickenhausen, Germany) and overlaid with mineral oil (M8410 Sigma). Images were captured on the stage of an inverted microscope (Eclipse 2000-U; Nikon, Düsseldorf, Germany) fitted with a spinning disk confocal unit (Ultra View RS3; Perkin-Elmer LAS, Jügesheim, Germany). A Nikon Plan Fluor 40X oil immersion lens (NA 1.30) was used. Twenty optical sections per blastocyst were captured using a Hamamatsu ORCA ER digital camera (Hamamatsu Photonics KK, Japan). Maximum intensity projections were analyzed with Image J Version 1.46j, counting the CDX2-positive, SOX17-positive and NANOG-positive cells manually.

Statistical analysis of morphometric data, gene expression data and developmental rates. ZP thickness, diameter of oocyte proper (vitellus), fetal rates and placental weights were analyzed by Wilcoxon using the statistical program JMP v.13 (SAS Institute Inc., USA). Developmental arrest at preimplantation stages and proportions of cell lineages in blastocysts were analyzed using the Fisher's Exact test. 


\section{References}

1 Engle, E. T. Pregnancy Following Super-Ovulation in the Mouse. Proceedings of the Society for Experimental Biology and Medicine 25 84-85 (1927).

2 Smith, P. E. \& Engle, E. T. Experimental evidence regarding the rôle of the anterior pituitary in the development and regulation of the genital system. American Journal of Anatomy 40, 159217 (1927).

3 Edwards, R. G. Historical significance of gonadotrophins in assisted reproduction. Reprod Biomed Online 10 Suppl 3, 3-10, doi:10.1016/s1472-6483(11)60384-2 (2005).

4 Fowler, R. E. \& Edwards, R. G. Induction of superovulation and pregnancy in mature mice by gonadotrophins. J Endocrinol 15, 374-384, doi:10.1677/joe.0.0150374 (1957).

5 Lunenfeld, B. Historical perspectives in gonadotrophin therapy. Hum Reprod Update 10, 453467, doi:10.1093/humupd/dmh044 (2004).

6 Behringer, R., Gertsenstein, M., Nagy, K. V. \& Nagy, A. Administration of Gonadotropins for Superovulation in Mice. Cold Spring Harb Protoc 2018, doi:10.1101/pdb.prot092403 (2018).

7 Li, L., Zheng, P. \& Dean, J. Maternal control of early mouse development. Development 137, 859870, doi:10.1242/dev.039487 (2010).

8 Mtango, N. R., Potireddy, S. \& Latham, K. E. Oocyte quality and maternal control of development. Int Rev Cell Mol Biol 268, 223-290, doi:10.1016/S1937-6448(08)00807-1 (2008).

9 Stitzel, M. L. \& Seydoux, G. Regulation of the oocyte-to-zygote transition. Science 316, 407-408, doi:10.1126/science.1138236 (2007).

10 Lee, M. et al. Adverse Effect of Superovulation Treatment on Maturation, Function and Ultrastructural Integrity of Murine Oocytes. Mol Cells 40, 558-566, doi:10.14348/molcells.2017.0058 (2017).

11 Champlin, A. K., Kuzia, S. J., Rice, B. A. \& Mobraaten, L. E. Cell surface characteristics of blastocysts from spontaneously ovulating and gonadotropin-treated mice. Biol Reprod 36, 439444, doi:10.1095/biolreprod36.2.439 (1987).

12 Elbling, L. Does gonadotrophin-induced ovulation in mice cause malformations in the offspring? Nature 246, 37-39, doi:10.1038/246037a0 (1973).

13 Elbling, L. Congenital malformations in mice after gonadotropin-induced ovulation. Proc Soc Exp Biol Med 149, 376-379, doi:10.3181/00379727-149-38811 (1975).

14 Lin, E., Li, Z., Huang, Y., Ru, G. \& He, P. High Dosages of Equine Chorionic Gonadotropin Exert Adverse Effects on the Developmental Competence of IVF-Derived Mouse Embryos and Cause Oxidative Stress-Induced Aneuploidy. Front Cell Dev Biol 8, 609290, doi:10.3389/fcell.2020.609290 (2020).

15 Nishimura, H. \& Shikata, A. The maldevelopment of the fetuses of mice treated with gonadotropic hormone before the conception. Okajimas Folia Anat Jpn 31, 195-202, doi:10.2535/ofaj1936.31.3-4_195 (1958).

16 Elbling, L. Malformations induced by hormones in mice and their transmission to the offspring. Exp Pathol (Jena) 11, 115-122, doi:10.1016/s0014-4908(75)80051-2 (1975).

17 Beaumont, H. M. \& Smith, A. F. Embryonic mortality during the pre- and post-implantation periods of pregnancy in mature mice after superovulation. J Reprod Fertil 45, 437-448, doi:10.1530/jrf.0.0450437 (1975).

18 Smith, C. M. \& Chrisman, C. L. Failure of exogenous gonadotrophin controlled ovulation to cause digit abnormalities in mice. Nature 253, 631-632, doi:10.1038/253631a0 (1975).

19 Diken, E. et al. Superovulation Influences Methylation Reprogramming and Delays Onset of DNA Replication in Both Pronuclei of Mouse Zygotes. Cytogenet Genome Res, doi:10.1159/000493779 (2018). 
20 Huffman, S. R., Pak, Y. \& Rivera, R. M. Superovulation induces alterations in the epigenome of zygotes, and results in differences in gene expression at the blastocyst stage in mice. Mol Reprod Dev 82, 207-217, doi:10.1002/mrd.22463 (2015).

21 Jahanbakhsh-Asl, E., Salehi, M., M., G.-N. \& Kato, Y. Superovulation Affects the Gene Expression Patterns of Mice Oocytes and Preimplantation Embryos Produced by Different Assisted Reproductive Technologies. INTERNATIONAL JOURNAL OF WOMEN'S HEALTH AND REPRODUCTION SCIENCES 6, 444-451 (2018).

22 Shi, W. \& Haaf, T. Aberrant methylation patterns at the two-cell stage as an indicator of early developmental failure. Mol Reprod Dev 63, 329-334, doi:10.1002/mrd.90016 (2002).

23 Uysal, F., Ozturk, S. \& Akkoyunlu, G. Superovulation alters DNA methyltransferase protein expression in mouse oocytes and early embryos. J Assist Reprod Genet 35, 503-513, doi:10.1007/s10815-017-1087-z (2018).

24 de Waal, E. et al. Gonadotropin stimulation contributes to an increased incidence of epimutations in ICSI-derived mice. Hum Mol Genet 21, 4460-4472, doi:10.1093/hmg/dds287 (2012).

25 Denomme, M. M., Zhang, L. \& Mann, M. R. Embryonic imprinting perturbations do not originate from superovulation-induced defects in DNA methylation acquisition. Fertil Steril 96, 734-738 e732, doi:10.1016/j.fertnstert.2011.06.055 (2011).

26 Saenz-de-Juano, M. D. et al. Genome-wide assessment of DNA methylation in mouse oocytes reveals effects associated with in vitro growth, superovulation, and sexual maturity. Clin Epigenetics 11, 197, doi:10.1186/s13148-019-0794-y (2019).

27 Huo, Y. et al. Single-cell DNA methylation sequencing reveals epigenetic alterations in mouse oocytes superovulated with different dosages of gonadotropins. Clin Epigenetics 12, 75, doi:10.1186/s13148-020-00866-w (2020).

28 Gahurova, L. et al. Transcription and chromatin determinants of de novo DNA methylation timing in oocytes. Epigenetics Chromatin 10, 25, doi:10.1186/s13072-017-0133-5 (2017).

29 Ertzeid, G. \& Storeng, R. The impact of ovarian stimulation on implantation and fetal development in mice. Hum Reprod 16, 221-225, doi:10.1093/humrep/16.2.221 (2001).

30 Van der Auwera, I., Pijnenborg, R. \& Koninckx, P. R. The influence of in-vitro culture versus stimulated and untreated oviductal environment on mouse embryo development and implantation. Hum Reprod 14, 2570-2574, doi:10.1093/humrep/14.10.2570 (1999).

31 Bonakdar, E. et al. A physiological, rather than a superovulated, post-implantation environment can attenuate the compromising effect of assisted reproductive techniques on gene expression in developing mice embryos. Mol Reprod Dev 82, 191-206, doi:10.1002/mrd.22461 (2015).

32 Deng, S., Xu, J., Zeng, J., Hu, L. \& Wu, Y. Ovarian stimulation leads to a severe implantation defect in mice. Reprod Biomed Online 27, 172-175, doi:10.1016/j.rbmo.2013.03.018 (2013).

33 Elmazar, M. M., Vogel, R. \& Spielmann, H. Maternal factors influencing development of embryos from mice superovulated with gonadotropins. Reprod Toxicol 3, 135-138, doi:10.1016/08906238(89)90047-6 (1989).

34 Fossum, G. T., Davidson, A. \& Paulson, R. J. Ovarian hyperstimulation inhibits embryo implantation in the mouse. J In Vitro Fert Embryo Transf 6, 7-10, doi:10.1007/BF01134574 (1989).

35 Mainigi, M. A. et al. Peri-implantation hormonal milieu: elucidating mechanisms of abnormal placentation and fetal growth. Biol Reprod 90, 26, doi:10.1095/biolreprod.113.110411 (2014).

36 Sibug, R. M., Helmerhorst, F. M., Tijssen, A. M., de Kloet, E. R. \& de Koning, J. Gonadotrophin stimulation reduces VEGF(120) expression in the mouse uterus during the peri-implantation period. Hum Reprod 17, 1643-1648, doi:10.1093/humrep/17.6.1643 (2002).

37 Weinerman, R., Ord, T., Bartolomei, M. S., Coutifaris, C. \& Mainigi, M. The superovulated environment, independent of embryo vitrification, results in low birthweight in a mouse model. Biol Reprod 97, 133-142, doi:10.1093/biolre/iox067 (2017). 
38 Sullivan-Pyke, C. et al. Timing of exposure to gonadotropins has differential effects on the conceptus: evidence from a mouse modeldagger. Biol Reprod 103, 854-865, doi:10.1093/biolre/ioaa109 (2020).

39 Edgar, D. H., Whalley, K. M. \& Mills, J. A. Preimplantation development following in vitro fertilization of mouse oocytes: effects of timing of superovulation and preincubation in vitro. $\mathrm{J} / \mathrm{n}$ Vitro Fert Embryo Transf 4, 111-115, doi:10.1007/BF01555450 (1987).

40 George, M. A. An analysis of the effect of the PMSG--HCG interval on the two-cell block. Hum Reprod 3, 249-250, doi:10.1093/oxfordjournals.humrep.a136687 (1988).

41 Zhang, X. et al. Ovarian stimulation retards postimplantation development and alters global gene expression profile of blastocysts in mouse. Fertil Steril 93, 2770-2773, doi:10.1016/j.fertnstert.2010.03.018 (2010).

42 Ozturk, S., Yaba-Ucar, A., Sozen, B., Mutlu, D. \& Demir, N. Superovulation alters embryonic poly(A)-binding protein (Epab) and poly(A)-binding protein, cytoplasmic 1 (Pabpc1) gene expression in mouse oocytes and early embryos. Reprod Fertil Dev 28, 375-383, doi:10.1071/RD14106 (2016).

43 Wetzels, A. M. et al. Gonadotropin hyperstimulation influences the 35S-methionine metabolism of mouse preimplantation embryos. J Assist Reprod Genet 12, 744-746, doi:10.1007/BF02212904 (1995).

44 Denomme, M. M. \& Mann, M. R. Maternal control of genomic imprint maintenance. Reprod Biomed Online 27, 629-636, doi:10.1016/j.rbmo.2013.06.004 (2013).

45 Israel, S., Casser, E., Drexler, H. C. A., Fuellen, G. \& Boiani, M. A framework for TRIM21-mediated protein depletion in early mouse embryos: recapitulation of Tead4 null phenotype over three days. BMC Genomics 20, 755, doi:10.1186/s12864-019-6106-2 (2019).

46 Israel, S. et al. An integrated genome-wide multi-omics analysis of gene expression dynamics in the preimplantation mouse embryo. Sci Rep 9, 13356, doi:10.1038/s41598-019-49817-3 (2019).

47 Pfeiffer, M. J. et al. Proteomic analysis of mouse oocytes reveals 28 candidate factors of the "reprogrammome". J Proteome Res 10, 2140-2153, doi:10.1021/pr100706k (2011).

48 Pfeiffer, M. J. et al. Differences in embryo quality are associated with differences in oocyte composition: a proteomic study in inbred mice. Proteomics 15, 675-687, doi:10.1002/pmic.201400334 (2015).

49 Schwarzer, C. et al. Maternal age effect on mouse oocytes: new biological insight from proteomic analysis. Reproduction 148, 55-72, doi:10.1530/REP-14-0126 (2014).

50 Wang, B., Pfeiffer, M. J., Drexler, H. C., Fuellen, G. \& Boiani, M. Proteomic Analysis of Mouse Oocytes Identifies PRMT7 as a Reprogramming Factor that Replaces SOX2 in the Induction of Pluripotent Stem Cells. J Proteome Res 15, 2407-2421, doi:10.1021/acs.jproteome.5b01083 (2016).

51 Chen, M. et al. Differential impacts of gonadotrophins, IVF and embryo culture on mouse blastocyst development. Reprod Biomed Online 39, 372-382, doi:10.1016/j.rbmo.2019.02.007 (2019).

52 Gao, L. L. et al. ZP3 is Required for Germinal Vesicle Breakdown in Mouse Oocyte Meiosis. Sci Rep 7, 41272, doi:10.1038/srep41272 (2017).

53 Bertrand, E., Van den Bergh, M. \& Englert, Y. Does zona pellucida thickness influence the fertilization rate? Hum Reprod 10, 1189-1193, doi:10.1093/oxfordjournals.humrep.a136116 (1995).

54 Saiz, N. \& Plusa, B. Early cell fate decisions in the mouse embryo. Reproduction 145, R65-80, doi:10.1530/REP-12-0381 (2013).

55 Yamanaka, Y., Lanner, F. \& Rossant, J. FGF signal-dependent segregation of primitive endoderm and epiblast in the mouse blastocyst. Development 137, 715-724, doi:10.1242/dev.043471 (2010).

56 Feldman, B., Poueymirou, W., Papaioannou, V. E., DeChiara, T. M. \& Goldfarb, M. Requirement of FGF-4 for postimplantation mouse development. Science 267, 246-249, doi:10.1126/science.7809630 (1995). 
57 Morris, S. A., Guo, Y. \& Zernicka-Goetz, M. Developmental plasticity is bound by pluripotency and the Fgf and Wnt signaling pathways. Cell Rep 2, 756-765, doi:10.1016/j.celrep.2012.08.029 (2012).

58 Christians, E. et al. Gene expression and chromatin organization during mouse oocyte growth. Dev Biol 207, 76-85, doi:10.1006/dbio.1998.9157 (1999).

59 Lin, Z. L. et al. Effect of anti-PMSG on distribution of estrogen receptor alpha and progesterone receptor in mouse ovary, oviduct and uterus. Zygote 23, 695-703, doi:10.1017/S0967199414000343 (2015).

60 Katagiri, S. et al. PMSG profiles in superovulated and anti-PMSG antiserum treated mice and heifers with enzymeimmunoassay. Jpn J Vet Res 39, 11-21 (1991).

61 Labrecque, R. \& Sirard, M. A. The study of mammalian oocyte competence by transcriptome analysis: progress and challenges. Mol Hum Reprod 20, 103-116, doi:10.1093/molehr/gat082 (2014).

62 Pan, H., O'Brien M, J., Wigglesworth, K., Eppig, J. J. \& Schultz, R. M. Transcript profiling during mouse oocyte development and the effect of gonadotropin priming and development in vitro. Dev Biol 286, 493-506, doi:10.1016/j.ydbio.2005.08.023 (2005).

63 Roller, R. J., Kinloch, R. A., Hiraoka, B. Y., Li, S. S. \& Wassarman, P. M. Gene expression during mammalian oogenesis and early embryogenesis: quantification of three messenger RNAs abundant in fully grown mouse oocytes. Development 106, 251-261 (1989).

64 Wang, Q. T. et al. A genome-wide study of gene activity reveals developmental signaling pathways in the preimplantation mouse embryo. Dev Cell 6, 133-144, doi:10.1016/s15345807(03)00404-0 (2004).

65 Coonrod, S. A. et al. Oocyte proteomics: localisation of mouse zona pellucida protein 3 to the plasma membrane of ovulated mouse eggs. Reprod Fertil Dev 16, 69-78, doi:10.10371/RD03079 (2004).

66 Qi, H. \& Wassarman, P. M. Secretion of zona pellucida glycoprotein mZP2 by growing oocytes from mZP3(+/+) and mZP3(-/-) mice. Dev Genet 25, 95-102, doi:10.1002/(SICI)15206408(1999)25:2<95::AID-DVG3>3.0.CO;2-6 (1999).

67 Zhao, M., Gold, L., Ginsberg, A. M., Liang, L. F. \& Dean, J. Conserved furin cleavage site not essential for secretion and integration of ZP3 into the extracellular egg coat of transgenic mice. Mol Cell Biol 22, 3111-3120, doi:10.1128/mcb.22.9.3111-3120.2002 (2002).

68 Bassil, R. et al. Can Oocyte Diameter Predict Embryo Quality? Reprod Sci 28, 904-908, doi:10.1007/s43032-020-00306-3 (2021).

69 Chan, P. J. Developmental potential of human oocytes according to zona pellucida thickness. J In Vitro Fert Embryo Transf 4, 237-241, doi:10.1007/BF01533763 (1987).

70 Marshall, K. L. \& Rivera, R. M. The effects of superovulation and reproductive aging on the epigenome of the oocyte and embryo. Mol Reprod Dev 85, 90-105, doi:10.1002/mrd.22951 (2018).

71 Wuri, L., Agca, C. \& Agca, Y. Morphometric, subcellular, in vitro fertilisation and embryonic developmental assessment of mouse oocytes produced by anti-inhibin serum or pregnant mare serum gonadotrophin superovulation. Reprod Fertil Dev 32, 474-483, doi:10.1071/RD19131 (2020).

72 Casser, E. et al. Differences in blastomere totipotency in 2-cell mouse embryos are a maternal trait mediated by asymmetric mRNA distribution. Mol Hum Reprod 25, 729-744, doi:10.1093/molehr/gaz051 (2019).

73 Artus, J. \& Chazaud, C. A close look at the mammalian blastocyst: epiblast and primitive endoderm formation. Cell Mol Life Sci 71, 3327-3338, doi:10.1007/s00018-014-1630-3 (2014).

74 Moore, R., Cai, K. Q., Tao, W., Smith, E. R. \& Xu, X. X. Differential requirement for Dab2 in the development of embryonic and extra-embryonic tissues. BMC Dev Biol 13, 39, doi:10.1186/1471-213X-13-39 (2013). 
75 Morris, S. M., Tallquist, M. D., Rock, C. O. \& Cooper, J. A. Dual roles for the Dab2 adaptor protein in embryonic development and kidney transport. EMBO J 21, 1555-1564, doi:10.1093/emboj/21.7.1555 (2002).

76 Tian, L. et al. Essential roles of Jab1 in cell survival, spontaneous DNA damage and DNA repair. Oncogene 29, 6125-6137, doi:10.1038/onc.2010.345 (2010).

77 Tomoda, K., Yoneda-Kato, N., Fukumoto, A., Yamanaka, S. \& Kato, J. Y. Multiple functions of Jab1 are required for early embryonic development and growth potential in mice. J Biol Chem 279, 43013-43018, doi:10.1074/jbc.M406559200 (2004).

78 Yang, D. H. et al. Disabled-2 is essential for endodermal cell positioning and structure formation during mouse embryogenesis. Dev Biol 251, 27-44, doi:10.1006/dbio.2002.0810 (2002).

79 Legge, M. \& Sellens, M. H. Optimization of superovulation in the reproductively mature mouse. J Assist Reprod Genet 11, 312-318, doi:10.1007/BF02215719 (1994).

80 Spindle, A. I. \& Goldstein, L. S. Induced ovulation in mature mice and developmental capacity of the embryos in vitro. J Reprod Fertil 44, 113-116, doi:10.1530/jrf.0.0440113 (1975).

81 Inyawilert, W., Liao, Y. J. \& Tang, P. C. Superovulation at a specific stage of the estrous cycle determines the reproductive performance in mice. Reprod Biol 16, 279-286, doi:10.1016/j.repbio.2016.10.004 (2016).

82 Lamas, S., Carvalheira, J., Gartner, F. \& Amorim, I. C57BL/6J mouse superovulation: schedule and age optimization to increase oocyte yield and reduce animal use. Zygote, 1-5, doi:10.1017/S0967199420000714 (2021).

83 Nelson, J. F., Karelus, K., Felicio, L. S. \& Johnson, T. E. Genetic influences on oestrous cyclicity in mice: evidence that cycle length and frequency are differentially regulated. J Reprod Fertil 94, 261-268, doi:10.1530/jrf.0.0940261 (1992).

84 Roldan, E. R., Vitullo, A. D., Merani, M. S. \& Von Lawzewitsch, I. Cross fertilization in vivo and in vitro between three species of vesper mice, Calomys (Rodentia, Cricetidae). J Exp Zool 233, 433442, doi:10.1002/jez.1402330312 (1985).

85 Sasamoto, S., Sato, K. \& Neito, H. Biological active life of PMSG in mice with special reference to follicular ability to ovulate. J Reprod Fertil 30, 371-379, doi:10.1530/jrf.0.0300371 (1972).

86 Bittner, A. K., Horsthemke, B., Winterhager, E. \& Grummer, R. Hormone-induced delayed ovulation affects early embryonic development. Fertil Steril 95, 2390-2394, doi:10.1016/j.fertnstert.2011.03.022 (2011).

87 Tavmergen, E., Goker, E. N., Sendag, F., Sendag, H. \& Levi, R. Comparison of short and long ovulation induction protocols used in ART applications according to the ovarian response and outcome of pregnancy. Arch Gynecol Obstet 266, 5-11, doi:10.1007/pl00007494 (2002).

88 Mak, W. et al. Natural cycle IVF reduces the risk of low birthweight infants compared with conventional stimulated IVF. Hum Reprod 31, 789-794, doi:10.1093/humrep/dew024 (2016).

89 Fatemi, H. M. et al. Cryopreserved-thawed human embryo transfer: spontaneous natural cycle is superior to human chorionic gonadotropin-induced natural cycle. Fertil Steril 94, 2054-2058, doi:10.1016/j.fertnstert.2009.11.036 (2010).

90 Hemkemeyer, S. A. et al. Effects of embryo culture media do not persist after implantation: a histological study in mice. Hum Reprod 29, 220-233, doi:10.1093/humrep/det411 (2014).

91 Weber, F. et al. Regulation of REM and Non-REM Sleep by Periaqueductal GABAergic Neurons. Nat Commun 9, 354, doi:10.1038/s41467-017-02765-w (2018).

92 Kilkenny, C., Browne, W. J., Cuthill, I. C., Emerson, M. \& Altman, D. G. Improving bioscience research reporting: the ARRIVE guidelines for reporting animal research. PLOS Biol 8, e1000412, doi:10.1371/journal.pbio.1000412 (2010).

93 Percie du Sert, N. et al. The ARRIVE guidelines 2.0: updated guidelines for reporting animal research. J Physio/ 598, 3793-3801, doi:10.1113/JP280389 (2020).

94 Parkening, T. A., Collins, T. J. \& Smith, E. R. Plasma and pituitary concentrations of LH, FSH and prolactin in aged female C57BL/6 mice. J Reprod Fertil 58, 377-386, doi:10.1530/jrf.0.0580377 (1980). 
95 Chatot, C. L., Ziomek, C. A., Bavister, B. D., Lewis, J. L. \& Torres, I. An improved culture medium supports development of random-bred 1-cell mouse embryos in vitro. J Reprod Fertil 86, 679688, doi:10.1530/jrf.0.0860679 (1989).

96 Chung, S. O. The thickness and volume change of the zona pellucida following ovulation and fertilization. Yonsei Med J 12, 28-33, doi:10.3349/ymj.1971.12.1.28 (1971).

97 Chung, S. O. Volume changes during the preimplantation stages of mouse egg development. Yonsei Med J 14, 63-90, doi:10.3349/ymj.1973.14.1.63 (1973).

98 Tartia, A. P. et al. Cell volume regulation is initiated in mouse oocytes after ovulation. Development 136, 2247-2254, doi:10.1242/dev.036756 (2009).

99 Ho, Y., Wigglesworth, K., Eppig, J. J. \& Schultz, R. M. Preimplantation development of mouse embryos in KSOM: augmentation by amino acids and analysis of gene expression. Mol Reprod Dev 41, 232-238, doi:10.1002/mrd.1080410214 (1995).

100 Graumann, J. et al. Stable isotope labeling by amino acids in cell culture (SILAC) and proteome quantitation of mouse embryonic stem cells to a depth of 5,111 proteins. Mol Cell Proteomics 7, 672-683, doi:10.1074/mcp.M700460-MCP200 (2008).

101 Gao, Y. et al. Protein Expression Landscape of Mouse Embryos during Pre-implantation Development. Cell Rep 21, 3957-3969, doi:10.1016/j.celrep.2017.11.111 (2017).

102 Alonso, A., Breuer, B., Steuer, B. \& Fischer, J. The F9-EC cell line as a model for the analysis of differentiation. Int J Dev Biol 35, 389-397 (1991).

103 Berstine, E. G., Hooper, M. L., Grandchamp, S. \& Ephrussi, B. Alkaline phosphatase activity in mouse teratoma. Proc Natl Acad Sci U S A 70, 3899-3903 (1973).

104 Pierce, G. B. Neoplasms, differentiations and mutations. Am J Pathol 77, 103-118 (1974).

105 Cox, J. \& Mann, M. MaxQuant enables high peptide identification rates, individualized p.p.b.range mass accuracies and proteome-wide protein quantification. Nat Biotechnol 26, 13671372, doi:10.1038/nbt.1511 (2008).

106 Shin, J. B. et al. Molecular architecture of the chick vestibular hair bundle. Nat Neurosci 16, 365374, doi:10.1038/nn.3312 (2013).

107 Anders, S. \& Huber, W. Differential expression analysis for sequence count data. Genome Biol 11, R106, doi:10.1186/gb-2010-11-10-r106 (2010).

108 Love, M. I., Huber, W. \& Anders, S. Moderated estimation of fold change and dispersion for RNA-seq data with DESeq2. Genome Biol 15, 550, doi:10.1186/s13059-014-0550-8 (2014).

109 Chen, E. Y. et al. Enrichr: interactive and collaborative HTML5 gene list enrichment analysis tool. BMC Bioinformatics 14, 128, doi:10.1186/1471-2105-14-128 (2013).

110 Kuleshov, M. V. et al. Enrichr: a comprehensive gene set enrichment analysis web server 2016 update. Nucleic Acids Res 44, W90-97, doi:10.1093/nar/gkw377 (2016).

111 Perez-Riverol, Y. et al. The PRIDE database and related tools and resources in 2019: improving support for quantification data. Nucleic Acids Res 47, D442-D450, doi:10.1093/nar/gky1106 (2019).

112 Vizcaino, J. A. et al. The PRoteomics IDEntifications (PRIDE) database and associated tools: status in 2013. Nucleic Acids Res 41, D1063-1069, doi:10.1093/nar/gks1262 (2013).

113 Schwarzer, C. et al. ART culture conditions change the probability of mouse embryo gestation through defined cellular and molecular responses. Hum Reprod 27, 2627-2640, doi:10.1093/humrep/des223 (2012).

114 Israel, S., Drexler, H. C. A., Fuellen, G. \& Boiani, M. The COP9 signalosome subunit 3 is necessary for early embryo survival by way of a stable protein deposit in mouse oocytes. Mol Hum Reprod, doi: 10.1093/molehr/gaab048 (in press) 


\section{Acknowledgements}

We thank the Max Planck Institute for Molecular Biomedicine and its Director, Prof. Hans R. Schöler, for infrastructural support. As part of it, we experienced outstanding support from the mouse housing facility, ensuring a dependable supply of mice needed to collect oocytes and embryos. We thank Annalen Nolte and Lina Lüken for technical help with the LC-MS/MS measurements. The microarray analysis was outsourced to the Core Genomic Facility of the Faculty of Medicine of the University of Muenster (Anika Witten, Andreas Huge). Critical reading of this manuscript by Verena Nordhoff and Ivan Bedzhov is gratefully acknowledged. This study was supported by the Deutsche Forschungsgemeinschaft (grant DFG BO-2540/4-3 to M.B., grant FU-583/5-1 to G.F. and grant TA 1076/1-1 to L.T.).

\section{Authors' contributions}

M.B. conceived and co-designed the study together with G.F. and L.T. S.I. collected the samples for proteome and transcriptome analysis, and performed the immunostainings. M.B. performed the embryo culture, the micromanipulations, the embryo transfers, analyzed the results, and wrote the manuscript together with L.T. L.T. performed the statistical analyses and wrote the manuscript together with M.B. L.T. and M.B. prepared the figures. G.F. contributed to the writing of the manuscript. H.D. performed the mass spectrometry analysis. W.M. and Y.S. provided the RNA sequencing. All authors approved the manuscript.

\section{Additional information}

Ethics declaration for human experiments and consent for publication

Not applicable

\section{Ethics declaration for animal experiments}


Mice were used for experiments according to the license issued by the Landesamt für Natur, Umwelt und Verbraucherschutz of the State of North Rhine-Westphalia, Germany (license number 81-02.04.2017.A432 and 84-02.04.2016.A229), in accordance with the procedures laid down in the European Directive 2010/63/EU. We observed the ARRIVE guidelines ${ }^{92,93}$ to the extent applicable, considering that the bulk of our experiments were conducted in vitro not in vivo, with the exception of embryo transfers.

\section{Availability of data and material}

All data underlying this article are available herein (Supplementary Tables) and in the following repositories. The raw data of Supplementary Table S1 (mass spectrometry) have been deposited to the ProteomeXchange Consortium via the PRIDE partner repository with the dataset identifier PXD021331 (username: reviewer_pxd021331@ebi.ac.uk; password: 4N7hyVLv). The raw data of Supplementary Table S2 (transcriptome analysis) have been deposited in the DNA Databank of Japan Sequence Read Archive with the dataset identifiers DRA005956 and DRA006335. An additional transcriptomic dataset is publicly available in NCBI's Gene Expression Omnibus and is accessible through GEO Series accession number GSE110599. The raw data of Supplementary Table S7 (mass spectrometry) have been deposited to the ProteomeXchange Consortium via the PRIDE partner repository with the dataset identifier PXD026347 (username: reviewer_pxd026347@ebi.ac.uk; password: cNouP1Bk).

\section{Competing interests}

The authors declare no competing interests.

\section{Figure legends}

Figure 1. Experimental design. B6C3F1 females were injected with gonadotropins (superovulation) or with vehicle (natural ovulation) and caged with vasectomized or with stud CD1 males to obtain MII or fertilized oocytes, respectively. These were removed at 8 a.m. on the day of the vaginal plug, and brought on equal footing by in vitro culture (IVC). Embryos were sampled in vitro at defined stages for molecular analysis, or were transplanted to naturally cycling females to generate fetuses. Abbreviations: eCG, equine chorionic gonadotropin; hCG, human chorionic gonadotropin; IVC, in vitro culture, MII, metaphase II. 
Figure 2. Expression analysis of the constitutively expressed $(n=2,844)$ proteome and their transcriptomic counterpart. (A, E) Hierarchical clustering of the proteome (A) and the transcriptome (E). Samples were clustered with the complete linkage algorithm, using a distance matrix based on the Pearson correlation coefficient computed between the expression values of the proteins. Proteins/transcripts are sorted according to pairwise Euclidean distances. (B-D and F-H) Principal component analysis. Each data point corresponds to a stage and treatment group. The first three principal components (PCs) of the data are represented. The PCA of the proteome resolves the superovulation and the natural ovulation groups in the $2^{\text {nd }}$ component, whereas the PCA of the transcriptome resolves the two groups in the $6^{\text {th }}$ component (not shown). Samples were derived from pools of 200-250 (proteome) and 210 (transcriptome) oocytes or embryos, in duplicate for each treatment.

Figure 3. Numbers of gonadotropin-sensitive proteins and their functional enrichment analysis. (A) Stagespecific number of proteins exhibiting a fold-change greater than or equal to $4,5,6,7$, or 8 (see scale) between the superovulation and natural ovulation groups. (B, C) Functional enrichment analysis of differentially expressed proteins using Enrichr. Only the ten terms with the smallest P-values are shown. Terms are sorted according to their combined scores, which are computed by taking the logarithm of the P-values and multiplying them by the z-scores of the deviation from the expected ranks. (B) Gene ontology terms of the altered proteome in the ontology 'Mammalian Phenotype'. (C) Gene ontology terms of the altered proteome in the ontology 'Biological process'.

Figure 4. Preimplantation phenotypes of superovulated and naturally ovulated oocytes. (A) The thickness of the ZP. Each data point corresponds to one oocyte. Wilcoxon test. (B) The diameter of the oocyte proper i.e. excluding zona and perivitelline space (vitellus). Each data point corresponds to one oocyte. Wilcoxon test. (C) The most advanced preimplantation stage reached in $\mathrm{KSOM}(\mathrm{aa})$ by the embryos of superovulated and naturally ovulated oocytes. Note that the scoring started from the 2-cell stage, since arrested 1-cells could be either oocytes which were not fertilized, or fertilized oocytes which failed to cleave. $\chi^{2}$ test. (D) Representative image of the triple immunostaining used to assign the blastocyst cells to the primary germ layers. (E-H) the cell numbers counted in the primary germ layers of blastocysts formed in $\mathrm{KSOM}(\mathrm{aa})$ by superovulated and naturally ovulated oocytes. Each data point corresponds to one blastocyst. Wilcoxon test. Abbreviations: $n$, number of; TE, trophectoderm; EPI, epiblast; pEND, primitive endoderm; ZP, zona pellucida.

Figure 5. Postimplantation performance of superovulated and naturally ovulated oocytes. Embryo transfers were conducted with at least 10 recipients per group relying on fixed numbers of 4-cell stage embryos ( $n=8$ per pseudopregnant female). These were transplanted to the oviduct, ensuring that i) the oocytes used were competent for cleavage, ii) time in culture was not unnecessarily long, iii) the later fetal densities in the uteri were balanced between the two experimental groups, and iv) undefined cues emanating from a stimulated genital tract were removed. (A) Number of implantations and (B) number of fetuses recorded at hysterectomy, 18 days after the transfer of 8 embryos to each pseudopregnant female. Each data point corresponds to one embryo transfer i.e. one mother. $P$ values refer to differences of numbers of implanted embryos or number of fetuses between groups (Wilcoxon test). (C) Birth weight and (D) placental weights of the individual fetuses. Each data point corresponds to one fetus or pup. $P$ values refer to differences of weight between groups (Wilcoxon test). Abbreviations: $n$, number of; $E T$, embryo transfer.

Figure 6. Effect of retrieving oocytes before or after the standard $48 \mathrm{~h}$ period of ovarian maturation. (A) The thickness of the ZP in MII oocytes derived from germinal vesicle oocytes prior to the ovulatory stimulus of hCG (IVM), compared to oocytes ovulated after the natural stimulus or after the exogenous stimulus ( $48 \mathrm{~h}$ vs $72 \mathrm{~h}$ ). Each data point corresponds to one oocyte. P-values refer to the differences of zona thickness between groups (Wilcoxon test). (B) Diameter of oocyte proper (vitellus) in the same oocytes as described in (A). Each data point corresponds to one oocyte. P-values refer to differences of oocyte diameter between groups (Wilcoxon test). (C) The most advanced preimplantation stage reached in $\operatorname{KSOM(aa)~by~the~embryos~of~superovulated~}$ oocytes that spent $48 \mathrm{~h}$ vs $72 \mathrm{~h}$ in the ovary, compared to naturally ovulated oocytes cultured. Note that the scoring started from the 2-cell stage, since arrested 1-cells could be either oocytes which were not fertilized, or fertilized oocytes which failed to cleave. $\chi^{2}$ test. (D) Number of fetuses recorded at hysterectomy, 18 days after the transfer of 8 embryos per pseudopregnant female. Each data point corresponds to one embryo transfer or mother. $\mathrm{P}$ values refer to differences of litter size between groups (Wilcoxon test). Abbreviations: IVM, in vitro maturation; NSN, non-surrounded nucleolus; SN, surrounded nucleolus; eCG, equine chorionic gonadotropin; ZP, zona pellucida; ET, embryo transfer. 
Figure 7. Proteome analysis (TMT) of oocytes superovulated with the $72 \mathrm{~h}$ regime compared to

conventionally superovulated, naturally ovulated or IVM oocytes. Hierarchical clustering of the proteome (A) and principal component analysis $(B, C, D)$. Each data point corresponds to one replicate. Oocytes superovulated with the $72 \mathrm{~h}$ regime form a separate subcluster $(A)$. The $1^{\text {st }}$ component of the PCA resolves the naturally ovulated oocytes from all the others $(B)$. The $2^{\text {nd }}$ component of the PCA resolves the IVM oocytes from all the others $(B, D)$. The $3^{\text {rd }}$ component of the PCA almost resolves the superovulated of the conventional stimulation protocol from the superovulated oocytes of the eCG $+72 \mathrm{~h}+\mathrm{hCG}$ protocol (C,D). Samples were derived from pools of 200-250 oocytes, in triplicate for each treatment (except IVM, in duplicate). Abbreviations: IVM, in vitro maturation; eCG, equine chorionic gonadotropin; hCG, human chorionic gonadotropin. 


\section{Supplementary Files}

This is a list of supplementary files associated with this preprint. Click to download.

- TahereratSupplementaryinformationJuly232021.pdf 